\title{
Relativistic Collapse Model With Tachyonic Features
}

\author{
Philip Pearle \\ Department of Physics, Hamilton College, Clinton, NY 13323
}

(June 3, 2022)

\begin{abstract}
A finite relativistic model for free particles, which describes the collapse of the statevector, is presented. The interaction of particles with a classical fluctuating field $w(x)$ causes collapse, as in the (so far) physically satisfactory nonrelativistic Continuous Spontaneous Localization (CSL) model. In previous relativistic models, where $w(x)$ has a white noise spectrum, collapse is accompanied by spontaneous creation of particles with all energies out of the vacuum (amounting to infinite energy/sec-vol). In this paper we explore one way of eliminating such vacuum excitation. We present a formalism which allows an arbitrary spectrum for $w(x)$. We point out, in lowest order of perturbation theory, that it is timelike components of this spectrum which are responsible for vacuum excitation but (appropriate, it seems, to a fundamentally nonlocal phenomenon) it is spacelike components which are responsible for collapse. We restrict the spectrum of $w(x)$ to that of a tachyon of mass $\mu=\hbar / a c \approx 1 \mathrm{eV}$, where $a \approx 10^{-5} \mathrm{~cm}$ is the Ghirardi-Rimini-Weber (GRW) collapse model scale parameter used in CSL. However, in higher orders than the first, there is still vacuum excitation due to the energy-momentum supplied by particle propagators. We opt to explore a simple means of eliminating this vacuum excitation: by removing the time-ordering operation from the statevector evolution so that particle propagators are on the mass-shell. The result is a finite theory: there is no vacuum excitation (and no need for renormalization) as each vertex describes a finite physical process, the spontaneous emission/absorption of a real tachyon by a particle (pair creation/annihilation is not permitted by energy-momentum conservation). This process may be thought of as analogous to the GRW "hitting" description of collapse. The cost of no time-ordering is that at each vertex a mass $M$ particle's wavefunction can spread by as much as $\approx c T(\mu / M)$ in time $T$. This has the result that, beginning at perturbation order $2 M / \mu$, there is a nonvanishing probability for the particle to be found outside its lightcone: for electrons and nucleons, the probability of this spacelike transport is negligibly small. Apart from this effect, the density matrix of particles in one region evolves independently of particles in another spacelike separated region. We thus obtain a finite, reasonably sensible, relativistic collapse model for free particles.
\end{abstract}

03.65.Bz,11.10.Lm,11.90.+t, 12.90.+b

\section{INTRODUCTION}

\section{A. CSL}

There is something missing in the foundations of standard quantum theory (SQT) which may motivate one to look for a possible deeper theory [1]. What SQT lacks is the specification of necessary conditions for when an event occurs. A sufficient condition is given: "upon completion of a measurement by an apparatus," after which one is supposed to "collapse the statevector" by hand to a state consistent with the outcome of the event. But this sufficient condition is ill-defined: what's a measurement? what's an apparatus? when is completion? do events occur in other circumstances?

In collapse models, this ill-defined sufficiency condition or "collapse postulate" of SQT is replaced by a well-defined evolution of the statevector. Collapse takes place dynamically, to a state consistent with the reality we observe around us, with a probability consistent with the predictions of SQT for most (but not all) experiments. This 30 year old program [2, 3] was reinvigorated by the Spontaneous Localization (SL) model of Ghirardi, Rimini and Weber [4, 5] (GRW) a decade ago. At present, the nonrelativistic Continuous Spontaneous Localization (CSL) model [6, 6 ] is the most satisfactory representative of this program.

The constraints on such a model are very severe. It must give the results of SQT for all experiments to date. It must generate a rapid collapse for a statevector which is a superposition of states of a macro-system (e.g., an apparatus) and the result of such a collapse must be a state which we actually observe in Nature. However, there should be negligible collapse of a statevector which is a superposition of states of a micro-system (e.g., of a particle undergoing interference). 
CSL consists of two equations. One is the Evolution Equation for the statevector. To Schrodinger's equation is added an extra term. It depends upon a classical fluctuating field $w(\mathbf{x}, t)$ which interacts with the number density of particles to cause collapse. This term contains two parameters introduced by GRW: $\lambda^{-1} \approx 10^{16} \sec \approx 300$ million years which characterizes the collapse time of an isolated nucleon and $a \approx 10^{-5} \mathrm{~cm}$ which characterizes the distance beyond which collapse is most effective. It also contains dimensionless parameters to characterize the coupling of $w(\mathbf{x}, t)$ to different particle types, e.g., nucleon and electron [8]. The second equation of CSL is a Probability Rule. It gives the probability that a particular $w(\mathbf{x}, t)$ is to be found in Nature.

Since the evolution of the statevector is different in CSL than in SQT, this allows for experimental tests. For example, a possible test is observation of a two slit interference pattern of a "large object" such as a $100^{\circ} \mathrm{A}$ sphere of mercury [9], since CSL predicts that the amplitudes of spatially separated packets will change with time [10] whereas SQT does not. While this has so far been too difficult to perform, another kind of test has been performed.

In CSL, collapse narrows wavefunctions, thereby increasing the energy of particles. This causes "spontaneous" excitation of bound states which then radiate 11, 12]. Experimental data on "spontaneous" radiation of X-rays in Ge, while not yet ruling out CSL or SQT, has recently been employed to provide valuable constraints on the parameters of CSL [13] 14]. It suggests that the coupling of $w(\mathbf{x}, t)$ to particles may be proportional to their mass, supporting the idea of a possible connection between collapse and gravity [15.16]. Also recently, it has been pointed out that experiments which place a limit on the lifetime of the proton could provide an even more stringent test [8], since the collapse mechanism ought to excite the quarks in the proton, and the decay products of the resulting unstable particle should be observed in proton lifetime experiments.

\section{B. Problems Of The First Relativistic Collapse Model}

Since quarks move highly relativistically, one must have a relativistic version of CSL—which we shall call RCSLin order to make a prediction of the effect of collapse on the proton lifetime.

Of course, this is not the only reason to construct a viable relativistic collapse model. After all, the relativistic symmetry exists in nature. John Bell was so concerned about the problem of making such a model that his first paper on collapse models [5] and his last paper [17] dealt with it. Collapse is a nonlocal behavior and it is not obvious how to make a nonlocal relativistic model, but in his first paper he argued that the nonrelativistic GRW model's mechanisms did not preclude a relativistic generalization.

Indeed, the subsequent discovery of CSL made it possible to construct an RCSL model $\left(\mathrm{RCSL}_{1}\right)$ [18 21]. But, in addition to collapsing the statevector, $\mathrm{RCSL}_{1}$ also has the undesirable feature of creating infinite energy/sec-vol (i.e., particles of all energies) out of the vacuum. In his last paper, Bell suggested that this difficulty indicates that RCSL 1 is not nonlocal enough, and he speculated that a resolution of the problem might eventually lead to something of fundamental significance.

The reason for this vacuum excitation is as follows. In $\mathrm{RCSL}_{1}$, a scalar field (of mass $\mu=a^{-1} \approx 1 \mathrm{eV}$ ) interacts as usual with the fermion field, and "dresses" each localized fermion with an average Yukawa field amplitude. Now, $w(\mathbf{x}, t)$ also interacts with this scalar field in such a way that the dynamics sees the statevector as a superposition of different scalar field amplitude states and attempts to collapse the statevector to one of these. When the statevector is a superposition of different localized fermion states, it is ipso facto a superposition of different scalar field amplitude "dresses" and the statevector is thereby collapsed toward one such state: this is the desired behavior.

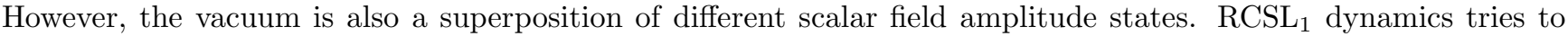
collapse the vacuum state to one of these. Such an alteration of the vacuum state amounts to exciting scalar particles out of the vacuum. The result is that the expectation value of the energy in each momentum mode of the scalar field increases linearly with time at the same constant slow rate of about $1 \mathrm{eV}$ per 300 million years. However, there are an infinite number of modes so there is infinite energy production. Indeed, in a relativistic theory, if any momentum mode of the vacuum is excited, then all must be excited. This means that a viable RCSL must have no vacuum excitation whatsoever.

\section{Outline Of Paper}

The purpose of this paper is to show one way of eliminating vacuum excitation and obtaining a finite RCSL model.

In $\mathrm{RCSL}_{1}, w(\mathbf{x}, t)$ has the statistics of white noise, so that its spectrum possesses all four-momenta in equal amounts. Although I introduced the idea of describing wavefunction collapse by a stochastic differential Schrodinger equation dependent upon white noise [3], this was largely for mathematical simplicity. White noise has all frequencies and physical processes do not, so I have always expected that eventually the spectrum of the noise would be modified. 
However, until this problem of vacuum excitation arose there had been no reason to make such a modification. A few years ago I proposed a formalism which generalizes CSL so that the spectrum of $w(\mathbf{x}, t)$ is adjustable [22]. The price paid is that the statevector's evolution is no longer Markovian (i.e., its future depends upon a past interval). Here we shall see that a relativistic version of this formalism allows removal of the culpable four-momenta which excite particles out of the vacuum. The result is a finite relativistic collapse model for free particles.

Section II contains a brief introduction to the formalism of CSL, and section II A gives an example of its use. In section III, the generalized formalism which allows $w(\mathbf{x}, t)$ to have a colored spectrum is introduced. In section IV, RCSL $_{1}$ and its generalized version are discussed and it is shown that the timelike part of the noise spectrum is responsible for producing particles out of the vacuum in lowest order of the perturbation theory expansion of the density matrix.

In section $\mathrm{V}$, this is cured by making the choice of a spacelike spectrum, that of a tachyon of mass $\mu\left(k^{2} \equiv k_{0}^{2}-\mathbf{k}^{2}=\right.$ $\left.-\mu^{2}\right)$. It is shown that the nonrelativistic $(c \rightarrow \infty)$ limit is CSL. It is also pointed out that the scalar field of RCSL 1 may be dispensed with without altering the physical content of the model if the theory just has $w(\mathbf{x}, t)$ directly interacting with the particle number density. At this point we have a model (which we call $\mathrm{RCSL}_{2}$ ) with no first order particle production from the vacuum, with sensible first order relativistic collapse behavior and with a sensible nonrelativistic limit.

However, in higher orders than the first there are still particles produced out of the vacuum. This is because these Feynman diagrams contain internal particle lines: the associated Feynman propagator can convey arbitrary four-momenta from a tachyon at a vertex to create particles. One way to eliminate this is to change the evolution equation by relativistically smearing the particle number density in such a way that the four-momenta each vertex allows propagators to exchange with a tachyon is limited. We shall purse that approach elsewhere, but here (in sections VI, VII and the remainder of this paper) we explore a simpler (in the sense of not introducing any new functions or constants) but perhaps more radical way of eliminating this vacuum excitation: by removing the timeordering operation from the evolution operator. This puts the propagator on the mass shell, thereby restricting its four-momenta and providing a completely finite collapse model (RCSL) to all orders. At each vertex, a real particle emits or absorbs a real tachyon. Pair production or annihilation is forbidden at each vertex by energy-momentum conservation, since a pair's timelike four-momentum cannot equal a tachyon's spacelike four-momentum. With no pair production, there is no particle production from the vacuum.

A big bonus is that there is no need for renormalization. All diagrams are finite as the physical process at each vertex is finite.

Section VIII A discusses no time-ordering, pointing out that a perturbation term may be thought of as involving successive forward and backwards-in-time evolutions (forward with interaction, backwards with free motion) of the statevector, which can lead to spacelike transport of particles. Section VIIIB shows how, in time T, the motion of a wavepacket in second order of perturbation theory can result in a particle of mass $M$ having a wavefunction spread out to $\approx c T(\mu / M)$ resulting, at order $2 M / \mu$, in a spread to distance $c T$ and, at higher orders, in a spread outside the particle's lightcone. However, because of the large order and the small phase space volume involved, the probability of this is extremely small. In section VIII we show that, with neglect of this effect, the model is local in the sense that particles evolve in one region independently of particles in a spacelike separated region.

In section [X] we give some results, the collapse rate and energy production rate for a free particle in lowest order.

It seems satisfying that, for the fundamentally nonlocal phenomenon of collapse, in order to eliminate vacuum excitation in relativistic models, we have been led to consider the fundamentally nonlocal relativistic entity: the tachyon.

In what follows, I have tried to make the body of the paper as compact and informative as possible, by putting the main argument in the text, and supportive calculations and discussion in appendices.

\section{CSL}

There are two major ideas behind CSL. One is the Gambler's Ruin mechanism for ensuring that the collapsed states occur with the probabilities of SQT [3]. The other is the GRW hitting mechanism for ensuring that the collapse rate is rapid for large objects and slow for small ones, and that the collapsed states are what we see around us, namely localized objects [何.

Consider the collapse dynamics of statevector $\left|\psi(t)>=a_{1}(t)\right| 1>+a_{2}(t) \mid 2>$ and, in particular, the behavior of the squared amplitudes $\left|a_{i}(t)\right|^{2}$. This is analogous to the behavior of the dollar amounts $d_{i}(t)$ in the Gambler's Ruin game, which is as follows. Suppose two gamblers start with $d_{1}(0), d_{2}(0)$ dollars respectively, where $d_{1}(0)+d_{2}(0)=100$, and they repeatedly toss a coin (analogous to the fluctuating field $w$ ). Depending on the outcome of the toss, one gives a dollar to the other, so the $d_{i}(t)$ fluctuate. Eventually one gambler wins, e.g., $d_{1}(t) / 100 \rightarrow 1$ and $d_{2}(t) \rightarrow 0$ and 
the game stops. It is easy to show that e.g., gambler 1 wins with probability $d_{1}(0) / 100$. With the correspondence $d_{i}(t) / 100 \rightarrow\left|a_{i}(t)\right|^{2}$, this means that the amplitudes $\left|a_{i}(t)\right|^{2}$ fluctuate, and eventually collapse occurs, e.g., $\mid \psi(t)>\rightarrow$ $1|1>+0| 2>$ with probability $\left|a_{1}(0)\right|^{2}$ : just the prediction of SQT!

In GRW's SL model [4,5] it is supposed that Nature subjects the wavefunction of a collection of particles not only to the usual Hamiltonian evolution, but also to random sudden alterations ("hits"). A hit is the multiplication of the wavefunction by a gaussian function $\exp -\left(\mathbf{x}_{n}-\mathbf{z}\right)^{2} / 2 a^{2}$, so that the part of the wavefunction which depends upon the nth particle's position $\mathbf{x}_{n}$ is suddenly narrowed to width $a$. The center of the gaussian, $\mathbf{z}$, is randomly chosen according to a certain Probability Rule which depends upon the wavefunction, and whose effect is to make it most likely that $\mathbf{z}$ is located where the wavefunction is largest. Such a hit occurs very infrequently on any one particle, at the rate $\lambda$. However, each particle is equally likely to be hit, and the wavefunction of particles belonging to an apparatus in a superposed state has particles correlated in such a way that one hit on one apparatus particle effects a collapse for the whole $\mathrm{N}$-particle apparatus, so that such a collapse occurs rapidly, in time $1 / \lambda N$.

In CSL [6,7] in effect a hit occurs every $\Delta t$ sec, except that the hit wavefunction (instead of replacing the original wavefunction) is multiplied by an amplitude $\sim \Delta t$ and added to the original wavefunction. In this way the wavefunction shape fluctuates, and the gambler's ruin dynamics is obtained. Also, the GRW hitting process destroys the (anti) symmetry of the wavefunction, and that problem is cured in CSL.

The CSL modified Schrodinger equation for the statevector $\mid \psi, t>_{w}$ which evolves under the influence of the fluctuating field $w$ is

$$
\frac{d \mid \psi, t>_{w}}{d t}=-i H\left|\psi, t>_{w}-\frac{1}{4 \lambda} \int d \mathbf{x}[w(\mathbf{x}, t)-2 \lambda A(\mathbf{x})]^{2}\right| \psi, t>
$$

Remarkably, this is a linear equation. All previous models had nonlinear equations, and it was suspected this had to be the case. It should be remarked that it is this linearity which makes relativistic generalizations easy. The operator $\mathrm{A}(\mathbf{x})$ is (apart from a factor $\sim a^{-3 / 2}$ ) essentially the number of particles in a volume $a^{3} \approx 10^{-15} \mathrm{~cm}^{3}$ centered around $\mathrm{x}$ :

$$
A(\mathbf{x}) \equiv \frac{1}{\left(\pi a^{2}\right)^{\frac{3}{4}}} \int d \mathbf{z} N(\mathbf{z}) e^{-\frac{(\mathbf{x}-\mathbf{z})^{2}}{2 a^{2}}}
$$

$\left(N(\mathbf{z}) \equiv \xi^{\dagger}(\mathbf{x}) \xi(\mathbf{x})\right.$ is the particle number density operator and $\xi(\mathbf{x})$ is the particle annihilation operator at $\left.\mathbf{x}\right)$.

CSL is completed by giving the probability density functional $P_{T}(w)$ that a particular realization of the field $w(\mathbf{x}, t)$ $(0 \leq t \leq T)$ occurs:

$$
P_{T}(w)={ }_{w}<\psi, T \mid \psi, T>{ }_{w}
$$

The statevector evolution (2.1) is nonunitary, so the norm of the statevector $\mid \psi, t>_{w}$ changes with time: Eq. (2.2) says that fields $w(\mathbf{x}, t)$ which lead to statevectors of largest norm are most likely. Conservation of probability is $\int D w P_{T}(w)=1$, where the functional integration element is $D w \sim \prod_{\mathbf{x}, t} d w(\mathbf{x}, t)$.

The solution of (2.1), in the "collapse interaction picture" where the statevector evolution is solely due to collapse dynamics, is

$$
\left|\psi, T>=\mathcal{T} e^{-\frac{1}{4 \lambda} \int_{T_{0}}^{T} d t d \mathbf{x}[w(\mathbf{x}, t)-2 \lambda A(\mathbf{x}, t)]^{2}}\right| \psi, T_{0}>
$$

where $\mathcal{T}$ is the time ordering operator and $A(\mathbf{x}, t) \equiv \exp i H t A(\mathbf{x}) \exp -i H t$.

Eq. (2.3) may also be written, using (2.1a), as

$$
\left|\psi, T>=\mathcal{T} e^{-\frac{1}{4 \lambda} \int_{T_{0}}^{T} d t d \mathbf{z} d \mathbf{z}^{\prime}\left[w^{\prime}(\mathbf{z}, t)-2 \lambda N(\mathbf{z}, t)\right] e^{-\frac{1}{4 a^{2}}\left(\mathbf{z}-\mathbf{z}^{\prime}\right)^{2}}\left[w^{\prime}\left(\mathbf{z}^{\prime}, t\right)-2 \lambda N\left(\mathbf{z}^{\prime}, t\right)\right]}\right| \psi, T_{0}>
$$

where $w^{\prime}$ is related to $w$ by $w(\mathbf{x}, t)=\int d \mathbf{z} w^{\prime}(\mathbf{z}, t)\left(\pi a^{2}\right)^{-\frac{3}{4}} \exp -(\mathbf{x}-\mathbf{z})^{2} / 2 a^{2}$ (we drop the prime hereafter). It is this form which will prove to be most valuable in section III for generalizing the statevector evolution.

\section{A. CSL Example}

To see how (2.4) leads to collapse for individual statevectors, let $H=0$ (so we are not concerned with how the usual dynamics competes with the collapse dynamics) and consider particles in a superposed state. Suppose that the 
initial statevector is $\left|\psi, T_{0}\right\rangle=\sum_{i} c_{i}\left|n_{i}\right\rangle$, where $N(\mathbf{x})\left|n_{i}\right\rangle=n_{i}(\mathbf{x})\left|n_{i}\right\rangle$, and the $n_{i}(\mathbf{x})$ represent distinctly different particle density distributions. Then Eqs. (2.4) and (2.2) become, respectively,

$$
\begin{gathered}
\left|\psi, T>=\sum_{i} c_{i}\right| n_{i}>e^{-\frac{1}{4 \lambda} \int_{T_{0}}^{T} d t d \mathbf{x} d \mathbf{x}^{\prime}\left[w(\mathbf{x}, t)-2 \lambda n_{i}(\mathbf{x})\right] e^{-\frac{1}{4 a^{2}}\left(\mathbf{x}-\mathbf{x}^{\prime}\right)^{2}}\left[w\left(\mathbf{x}^{\prime}, t\right)-2 \lambda n_{i}\left(\mathbf{x}^{\prime}\right)\right]} \\
P_{T}(w)=\sum_{i}\left|c_{i}\right|^{2} e^{-\frac{1}{2 \lambda} \int_{T_{0}}^{T} d t d \mathbf{x} d \mathbf{x}^{\prime}\left[w(\mathbf{x}, t)-2 \lambda n_{i}(\mathbf{x})\right] e^{-\frac{1}{4 a^{2}\left(\mathbf{x}-\mathbf{x}^{\prime}\right)^{2}}\left[w\left(\mathbf{x}^{\prime}, t\right)-2 \lambda n_{i}\left(\mathbf{x}^{\prime}\right)\right]}}
\end{gathered}
$$

As $T$ increases, any $w(\mathbf{x}, t)$ which makes one term in the sum in Eq. (2.5a) or (2.5b) large makes the other terms small. For example, suppose that $w(\mathbf{x}, t)=2 \lambda n_{i}(\mathbf{x})$ : then, while the $i$ th exponential $=1$, the $j$ th exponential in (2.5a) has the value

$$
e^{-\lambda\left(T-T_{0}\right) \int d \mathbf{x} d \mathbf{x}^{\prime}\left[n_{i}(\mathbf{x})-n_{j}(\mathbf{x})\right] e^{-\frac{1}{4 a^{2}}\left(\mathbf{x}-\mathbf{x}^{\prime}\right)^{2}}\left[n_{i}\left(\mathbf{x}^{\prime}\right)-n_{j}\left(\mathbf{x}^{\prime}\right)\right]}
$$

which approaches 0 for large $T$. This is also the behavior if, more generally, $w(\mathbf{x}, t)$ almost always fluctuates uniformly about $2 \lambda n_{i}(\mathbf{x})$ (for all other behaviors of $w$, all the terms in (2.5) asymptotically vanish). Thus the terms in Eqs. (2.5) evolve toward regions of disjoint support in $w$-space When $w$ lies in the $i$ th region, $|\psi, T>\rightarrow \sim| n_{i}>$ according to (2.5a), while the integrated probability over the $i$ th region $\int D w P(w) \rightarrow\left|c_{i}\right|^{2}$ according to (2.5b).

The density matrix which follows from Eqs. (2.2), 2.3) is

$$
\begin{aligned}
\rho(T) \equiv & \int D w P(w) \frac{|\psi, T><\psi, T|}{<\psi, T \mid \psi, T>} \\
& =\mathcal{T} e^{-\frac{\lambda}{2} \int_{T_{0}}^{T} d t d \mathbf{x} d \mathbf{x}^{\prime}[N(\mathbf{x}, t) \otimes 1-1 \otimes N(\mathbf{x}, t)] e^{-\frac{1}{4 a^{2}\left(\mathbf{x}^{\prime} \mathbf{x}^{\prime}\right)^{2}}\left[N\left(\mathbf{x}^{\prime}, t\right) \otimes 1-1 \otimes N\left(\mathbf{x}^{\prime}, t\right)\right]} \rho\left(T_{0}\right)}
\end{aligned}
$$

We are employing the notation $A \otimes B \rho=A \rho B$, and $\mathcal{T}$ time-reverses operators to the right of $\rho\left(T_{0}\right)$.

The collapse behavior of the previous example may easily be seen in the decay of $\rho(T)$ 's off-diagonal density matrix elements:



As a simple example, suppose $\left|n_{1}\right\rangle$ and $\mid n_{2}>$ each describe a clump of $\mathrm{N}$ particles with dimensions $<<a$, but with centers of mass of the two states at a distance $>>a$ apart. Then the gaussian in Eq. $(2.8) \approx 1$ if $\mathbf{x}, \mathbf{x}^{\prime}$ are both located in region 1 (or both in 2) and $\approx 0$ if $\mathbf{x}, \mathbf{x}^{\prime}$ are located in regions 1 and 2 respectively. Therefore, Eq. (2.8) yields

$$
<n_{1}|\rho(T)| n_{2}>=c_{1} c_{2}^{*} e^{-\lambda N^{2}\left(T-T_{0}\right)}
$$

illustrating how the collapse rate is large for a superposition of states describing a large number of particles in different locations.

It should be emphasized that the dynamics of an individual statevector under a probable $w(x)$ allows nonlocal influences whose outcome, however, is not controllable. For example, a particle whose wavefunction describes it as in an equal superposition of "here" plus "there" may be voluntarily entangled with a position measuring apparatus located near "here," by turning the apparatus on to measure whether the particle is "here". As a result, for half the probable $w(x)$ 's, the wavepacket "there" will disappear, and for the other half it will grow to total probability 1. On the other hand, because the field $w(x)$ which determines the outcome is not controllable, for the ensemble of statevectors evolving under all possible $w(x)$ 's, no nonlocal communication is possible. The proof of this for RCSL is given in section VIIIC: the comparable proof for CSL is essentially the same

\section{GENERALIZED FORMALISM}

We now postulate, as a generalization of the CSL evolution Eq. (2.4), the statevector evolution

$$
\left|\psi, T>=\mathcal{T} e^{-\frac{1}{4 \gamma} \int_{T_{0}}^{T} d x d x^{\prime}[w(x)-2 \gamma F(x)] G\left(x-x^{\prime}\right)\left[w\left(x^{\prime}\right)-2 \gamma F\left(x^{\prime}\right)\right]}\right| \psi, T_{0}>
$$


In Eq. (3.1), $x \equiv(t, \mathbf{x}), d x \equiv d t d \mathbf{x}, F(x)$ is a family of operators which commute at equal times, and $G\left(x-x^{\prime}\right)$ is a positive definite even function, i.e., its Fourier transform $\tilde{G}(k)$ is real, even and $\geq 0$. In addition, we retain the probability rule (2.2).

Eq. (3.1) reduces to the CSL evolution Eq. (2.4) when

$$
\begin{aligned}
\gamma & =\lambda \\
G\left(x-x^{\prime}\right) & =\delta\left(t-t^{\prime}\right) e^{-\left(\mathbf{x}-\mathbf{x}^{\prime}\right)^{2} / 4 a^{2}} \\
F\left(\mathbf{x}, T_{0}\right) & \equiv N(\mathbf{x}) \\
F(x) & =e^{i H t} F(\mathbf{x}) e^{-i H t}
\end{aligned}
$$

If $G\left(x-x^{\prime}\right) \sim \delta\left(t-t^{\prime}\right)$, as in Eq. (3.2b), then $w(\mathbf{x}, t)$ is uncorrelated at different times and $\mid \psi, T>$ satisfies a stochastic differential equation (e.g. Eq. (2.1)), but this is not so in general.

When $G\left(x-x^{\prime}\right) \nsim \delta\left(t-t^{\prime}\right)$, then $w(\mathbf{x}, t)$ is correlated at different times and the statevector evolution is no longer

Markovian. In particular, the Markovian identity $\int_{T}^{T^{\prime}} D w P_{T^{\prime}}(w)=P_{T}(w)$ enjoyed by CSL no longer holds. We take this to mean that the probability estimate of $w(\mathbf{x}, t)$ (regarded as the best measure possible based upon limited information, namely lack of knowledge of the future values of $\mathrm{w}$ [23]) improves with time: strictly speaking, only $P_{\infty}(w)$ gives the correct probability of $w$. However, for $G$ utilized in this paper, $P_{T}(w)$ essentially changes negligibly with increasing $\mathrm{T}$.

That this generalized formalism produces collapse for individual statevectors is shown in Appendix A.

The density matrix which follows from (3.1), 2.2) is

$$
\begin{aligned}
\rho(T) & \equiv \int D w P_{T}(w) \frac{|\psi, T><\psi, T|}{<\psi, T \mid \psi, T>} \\
& =\mathcal{T} e^{-\frac{\gamma}{2} \int_{T_{0}}^{T} d x d x^{\prime}[F(x) \otimes 1-1 \otimes F(x)] G\left(x-x^{\prime}\right)\left[F\left(x^{\prime}\right) \otimes 1-1 \otimes F\left(x^{\prime}\right)\right]} \rho\left(T_{0}\right)
\end{aligned}
$$

It is useful and illuminating to write the statevector (3.1) and density matrix (3.3) as Fourier transforms (Appendix B):

$$
\begin{aligned}
& \left|\psi, T>=\mathcal{T} \int D \eta e^{-\gamma \int_{-\infty}^{\infty} d x d x^{\prime} \eta(x) G^{-1}\left(x-x^{\prime}\right) \eta\left(x^{\prime}\right)} e^{i \int_{T_{0}}^{T} d x \eta(x)[w(x)-2 \gamma F(x)]}\right| \psi, T_{0}> \\
& \rho(T)=\mathcal{T} \int D \eta e^{-2 \gamma \int_{-\infty}^{\infty} d x d x^{\prime} \eta(x) G^{-1}\left(x-x^{\prime}\right) \eta\left(x^{\prime}\right)} e^{-i 2 \gamma \int_{T_{0}}^{T} d x \eta(x)[F(x) \otimes 1-1 \otimes F(x)]} \rho\left(T_{0}\right)
\end{aligned}
$$

This shows that the statevector and density matrix may be written as superpositions of unitary evolutions $\exp -i 2 \gamma \int_{T_{0}}^{T} d x \eta(x) F(x)$, describing the interaction of a classical "noise" field $\eta(x)$ with the operator $F(x)$. (The superposition has a Gaussian weight and, in the case of Eq. (3.4), also a phase factor weight.) Thus it is actually the spectrum of $\eta(x)$ (the inverse of the spectrum of $w(x)$ ) which excites the quantum system [22]. Eq. (3.5) illustrates the well known fact that the density matrix arising from an ensemble of "true collapse" evolutions (as discussed here) can be equal to a density matrix obtained from an ensemble of non-collapse unitary evolutions.

Eq. (3.5) is particularly useful for calculations because it enables one to employ Feynman techniques in evaluating the perturbation expansion of the density matrix.



We now apply this formalism to a specific relativistic model.

In Eq. (3.1) let $F(x)=\phi(x)$ be a scalar field of mass $\mu$, and also let the Hamiltonian $H$ be such that $\phi(x)$ "dresses" a particle of mass $M$. The "dress" coupling should be $g \phi(x): \bar{\psi}(x) \psi(x)$ :, where $\psi(x)$ is a Dirac fermion field, but I do not wish to clutter up the calculations with Dirac algebra. Therefore I shall use a boson particle $\psi(x)$, with coupling $g(2 M) \phi(x): \psi(x) \psi(x)$ : (the factor $(2 M)$ keeps $g$ dimensionless). In the usual interaction picture (fields obey free-field evolution) the density matrix (3.5) is

$$
\begin{gathered}
\rho(\sigma)=\mathcal{T} \int D \eta e^{-2 \gamma \int_{-\infty}^{\infty} d x d x^{\prime} \eta(x) G^{-1}\left(x-x^{\prime}\right) \eta\left(x^{\prime}\right)} e^{-i 2 \gamma \int_{\sigma_{0}}^{\sigma} d x \eta(x)[\phi(x) \otimes 1-1 \otimes \phi(x)]} \\
\cdot e^{-i g(2 M) \int_{\sigma_{0}}^{\sigma} d x\left[\phi(x): \psi^{2}(x): \otimes 1-1 \otimes \phi(x): \psi^{2}(x):\right]} \rho\left(\sigma_{0}\right)
\end{gathered}
$$


In Eq. (4.1), $\left(\sigma_{0}, \sigma\right)$ are arbitrary nonintersecting initial and final spacelike hypersurfaces (which will be replaced by $(-T / 2, T / 2)$ for calculations), and $G\left(x-x^{\prime}\right)$ is a Lorentz scalar:

$$
G\left(x-x^{\prime}\right)=\frac{1}{(2 \pi)^{4}} \int d k e^{i k \cdot\left(x-x^{\prime}\right)} \tilde{G}\left(k^{2}\right)
$$

where $\tilde{G}\left(k^{2}\right)$ is real and positive.

It is shown in Appendix $\mathrm{C}$ that such a model is Lorentz invariant from both the passive point of view (i.e., the description of the evolution of the statevector $\left|\psi, \sigma_{0}\right\rangle$ to $|\psi, \sigma\rangle$ is the same from all Lorentz frames) and the active point of view (i.e., if $\left|\psi, \sigma_{0}\right\rangle$ evolves into $|\psi, \sigma\rangle$ then the Lorentz transform of $\left|\psi, \sigma_{0}\right\rangle$ evolves into the Lorentz transform of $|\psi, \sigma\rangle)$.

$\mathrm{RCSL}_{1}$ is the (Markovian) model obtained with $G\left(x-x^{\prime}\right)=\delta\left(x-x^{\prime}\right)$, i.e., $\tilde{G}\left(k^{2}\right)=1$. The qualitative collapse behavior of this relativistic model (which is the same as in all the rest of the models presented here) has been discussed extensively 19,24 26], especially by Ghirardi and Grassi, and we shall not go over that ground in any detail here. The main feature is that the picture of the collapse process is hypersurface dependent (e.g., in the example at the end of section II A, the spacetime region where the wavepacket "there" disappears depends upon the reference frame from which the evolution is described). Nonetheless, this feature is experimentally consistent as "collapse time" and "collapse location" are not measurable quantities, and all observers agree on what is measurable, the collapse result. Some of this behavior was presaged in their discussion of the collapse postulate in relativistic quantum theory by Aharonov and Albert 27].

We shall now discuss the calculation of the energy produced from the vacuum to (lowest) order $\gamma$ : with no extra labor we can consider $\mathrm{RCSL}_{1}$ together with the more general case where $\tilde{G}\left(k^{2}\right)$ does not have to equal 1 . It is useful to introduce Feynman diagrams to discuss the perturbation expansion of the density matrix (4.1). The diagrammatic representation of the two vertices, $2 \gamma \eta(x) \phi(x)$ and $g(2 M) \phi(x): \psi^{2}(x)$ :, are illustrated in Figs. 1a, $1 \mathrm{~b}$.

After performing the perturbation expansion in powers of $\gamma$ and $g$, we must integrate over $\eta$ using, for example,

$$
\begin{gathered}
\int D \eta e^{-2 \gamma \int_{-\infty}^{\infty} d x d x^{\prime} \eta(x) G^{-1}\left(x-x^{\prime}\right) \eta\left(x^{\prime}\right)} 2 \gamma \eta(z)=0 \\
\int D \eta e^{-2 \gamma \int_{-\infty}^{\infty} d x d x^{\prime} \eta(x) G^{-1}\left(x-x^{\prime}\right) \eta\left(x^{\prime}\right)} 2 \gamma \eta(y) 2 \gamma \eta(z)=\gamma G(y-z)
\end{gathered}
$$

Odd powers of $\gamma \eta$ give 0 , while even powers give products of factors $\gamma G\left(x_{i}-x_{j}\right)$, with all possible permutations of the indices. Thus all vertices containing $\eta$ are "paired up," and may be regarded as connected by a "noise propagator" $\gamma G\left(x_{i}-x_{j}\right)$. The symbol for the noise propagator is a thick line, which is illustrated connecting two $\eta$ vertices in Fig. 1c. In momentum space, the noise propagator contributes a factor $\gamma \tilde{G}\left(k^{2}\right)$.

Because it is a density matrix we are expanding, we get terms to the left of $\rho\left(T_{0}\right)$ and terms to the right of $\rho\left(T_{0}\right)$. In the diagrams we draw, these shall be separated by a vertical dotted line. The only thing that can cross the line is a noise propagator. We note that operators to the right of $\rho\left(T_{0}\right)$ are time-reversed. This means that the four-momenta may be considered as going backwards in time to the right of the dotted line (or alternatively, we may consider that they are negative four-momenta going forward in time).

In $\mathrm{RCSL}_{1}$, particles are produced from the vacuum. We shall now see that this may be avoided to order $\gamma$ if $\tilde{G}\left(k^{2}\right)$ vanishes for timelike $k^{2}$. The diagrams which describe the evolution of the vacuum to order $\gamma$ are shown in Figs. 2a,b,c. Diagrams 2a,b describe the decrease of the vacuum state amplitude due to $\phi$-particle creation, while 2c describes creation of a $\phi$-particle. The contribution of these three diagrams is calculated in Appendix D. For diagram 2c, which is connected across the dotted line, four-momentum goes in at the right and comes out at the left: in this way it is possible, from the diagrammatic point of view, to have creation of a $\phi$-particle out of the vacuum and still conserve four-momentum. From the physical point of view, of course, it is the four-momentum in the noise that generates the $\phi$-particle.

The result of the calculation, Eq. (D4), is that the average created energy/sec-vol is proportional to $\tilde{G}\left(\mu^{2}\right)$ (and infinite, because it is also proportional to $\left.\int d \mathbf{k}\right)$. Thus, if $\tilde{G}\left(\mu^{2}\right)=0$, then there is no $\phi$-particle production out of the vacuum to order $\gamma$. Indeed, then there is no $\phi$-particle production out of the vacuum to order $\gamma^{n}$ (arbitrary $n$ ), since all $\phi$-particle creation diagrams not involving $g$ consist of disconnected diagrams of type $2 \mathrm{c}$, or such diagrams with the noise propagator replaced by alternating noise propagators and $\phi$ propagators.

However, there is still particle pair production out of the vacuum to order $\gamma g^{2}$. The relevant diagram is shown in Fig. 2d. Its contribution is 


$$
\sim \gamma g^{2} \frac{\tilde{G}\left[\left(p_{1}+p_{2}\right)^{2}\right]}{\left[\left(p_{1}+p_{2}\right)^{2}-\mu^{2}\right]^{2}}
$$

where $p_{1}, p_{2}$ label the outgoing particles. To prevent this, $\tilde{G}\left(k^{2}\right)$ must vanish for $k^{2} \geq(2 M)^{2}$.

Now, we may assume that $w(\mathbf{x}, t)$ can be coupled to any particle with nonzero mass [8], e.g., an electron, or a neutrino (if it should turn out to have mass). Thus the condition of no vacuum excitation to order $\gamma g^{2}$ leaves very little room on the $k^{2} \geq 0$ line where $\tilde{G}\left(k^{2}\right)$ does not vanish. In fact, next consider $\phi$-particle pair production to order $\gamma g^{6}$, contributed by the diagram (2e). For this to vanish, $\tilde{G}\left(k^{2}\right)$ must vanish for $k^{2} \geq(2 \mu)^{2} \approx(2 \mathrm{eV})^{2}$. Finally, suppose the particle is charged, so it is coupled to the electromagnetic field. A diagram like 2e, with the created $\phi$-particles replaced by photons, shows that we must have $\tilde{G}\left(k^{2}\right) \equiv 0$ for $k^{2} \geq 0$ to prevent photon production to order $\sim \gamma$.

Thus to prevent particles from being created out of the vacuum at an infinite rate/vol to an order $\sim \gamma$ we must let $\tilde{G}\left(k^{2}\right) \neq 0$ only for spacelike $k^{2}$.

\section{RCSL 2}

Does restricting $\tilde{G}\left(k^{2}\right)$ to be nonvanishing only for $k^{2}<0$ make collapse behavior untenable? On the contrary, as we shall see, $\tilde{G}\left(k^{2}\right)$ for $k^{2} \geq 0$ is irrelevant for collapse.

Consider the behavior of $G\left(x-x^{\prime}\right)$ in the limit $c \rightarrow \infty$ : this is one way of looking at the nonrelativistic limit. Use of Eq. (4.2) with the $c$ dependence explicitly displayed results in

$$
\begin{aligned}
G\left(\mathbf{x}-\mathbf{x}^{\prime}, t-t^{\prime}\right) & =\frac{1}{(2 \pi)^{4}} \int d \omega d \mathbf{k} e^{i \omega\left(t-t^{\prime}\right)-i \mathbf{k} \cdot\left(\mathbf{x}-\mathbf{x}^{\prime}\right)} \tilde{G}\left(\frac{\omega^{2}}{c^{2}}-\mathbf{k}^{2}\right) \\
& \longrightarrow \delta\left(t-t^{\prime}\right) \frac{1}{(2 \pi)^{3}} \int d \mathbf{k} e^{-i \mathbf{k} \cdot\left(\mathbf{x}-\mathbf{x}^{\prime}\right)} \tilde{G}\left(-\mathbf{k}^{2}\right)
\end{aligned}
$$

Thus it is only negative values of $k^{2}$ which are relevant arguments of $\tilde{G}$ in this limit.

We note that the time dependence of $G$ in this limit is $\sim \delta\left(t-t^{\prime}\right)$, identical to that of CSL. What about the spatial behavior of the limit (5.1)? In the rest of this paper we shall make the simplest choice of spacelike spectrum

$$
\tilde{G}\left(k^{2}\right)=\delta\left(k_{0}^{2}-\mathbf{k}^{2}+\mu^{2}\right)
$$

corresponding to a tachyon of mass $\mu=a^{-1} \approx 1 \mathrm{eV}$. With this choice, Eq. (5.1) becomes

$$
G\left(\mathbf{x}-\mathbf{x}^{\prime}, t-t^{\prime}\right) \longrightarrow \delta\left(t-t^{\prime}\right) \frac{1}{(2 \pi)^{2}} \frac{\sin \left[\left|\mathbf{x}-\mathbf{x}^{\prime}\right| / a\right]}{\left|\mathbf{x}-\mathbf{x}^{\prime}\right|}
$$

The spatial dependence of $G$ in Eq. (5.3) is quite a satisfactory replacement [28] for the Gaussian dependence (3.2b]) introduced by GRW and which is usually employed in CSL. 29

It is worth noting here the exact solution for $G$ of which (5.3) is the $c \rightarrow \infty$ limit. Putting (5.2) into (4.2) yields

$$
\begin{aligned}
G\left(x-x^{\prime}\right) & =-\frac{1}{8 \pi^{2} a\left|x-x^{\prime}\right|} N_{1}\left(\left|x-x^{\prime}\right| / a\right) & & \left(x-x^{\prime}\right)^{2}<0 \\
& =-\frac{1}{4 \pi^{3} a\left|x-x^{\prime}\right|} K_{1}\left(\left|x-x^{\prime}\right| / a\right) & & \left(x-x^{\prime}\right)^{2}>0
\end{aligned}
$$

$\left(\left|x-x^{\prime}\right| \equiv \sqrt{\left|\left(x-x^{\prime}\right)^{2}\right|}\right.$ and $N, K$ are Bessel Functions). For spacelike $x-x^{\prime}, G$ oscillates on the scale $a \approx 10^{-5} \mathrm{~cm}$ (and decreases as $\left|x-x^{\prime}\right|^{-\frac{3}{2}}$ ), while for timelike $x-x^{\prime}, G$ decays exponentially with time constant $a / c \approx 10^{-15}$ sec.

For $\mathrm{RCSL}_{1}$, where $\tilde{G}\left(k^{2}\right)=1$, the spatial scale of collapse is not set by G: rather it is set by the $\phi$-mass which governs the spatial extent of the Yukawa dressing of the particles. With the same mass chosen for the tachyon, this physical mechanism for the scale appears to be - and is - redundant: the $\phi$-particle is no longer needed.

To see this, compare the RCSL 1 diagrams in Fig. 3 with their counterpart diagrams in Fig. 4, belonging to what we shall call $\mathrm{RCSL}_{2}$. In each $\mathrm{RCSL}_{1}$ diagram, two $\phi$-propagators are attached to the ends of a noise propagator, providing a factor $\sim \gamma \tilde{G}\left(k^{2}\right)\left(k^{2}-\mu^{2}\right)^{-2}=\gamma \delta\left(k^{2}+\mu^{2}\right)\left(-2 \mu^{2}\right)^{-2}$. Thus the $\phi$-propagators just provide a numerical factor (and would do so even were the $\phi$ mass not $\mu$ ) which may be incorporated into $\gamma$. Therefore the $\phi$ particles 
may be completely dispensed with. (We note that external $\phi$-lines which show up in higher order diagrams of RCSL 1 , describing production, absorption or scattering of $\phi$ particles, are not needed for collapse behavior.)

The new model, $\mathrm{RCSL}_{2}$, is described by the statevector evolution Eq. (3.1) with $G\left(x-x^{\prime}\right)$ given by Eqs. (4.2), (5.2) and with $F(x)=2 M: \psi^{2}(x)$ : 30]. The associated density matrix is

$$
\rho(\sigma)=\mathcal{T} \int D \eta e^{-2 \gamma \int_{-\infty}^{\infty} d x d x^{\prime} \eta(x) G^{-1}\left(x-x^{\prime}\right) \eta\left(x^{\prime}\right)} e^{-i 2 \gamma(2 M) \int_{\sigma_{0}}^{\sigma} d x \eta(x)\left[: \psi^{2}(x): \otimes 1-1 \otimes: \psi^{2}(x):\right]} \rho\left(\sigma_{0}\right)
$$

The coupling constant $\gamma$ is dimensionless. In the nonrelativistic limit discussed above and with neglect of pair creation/annihilation (replacement of : $\psi^{2}(x)$ : by $2 \xi^{\dagger}(x) \xi(x) / 2 M$ ), it is seen that the nonrelativistic limit of $\mathrm{RCSL}_{2}$ is CSL.

\section{DENSITY MATRIX TO LOWEST ORDER IN RCSL 2}

The perturbation series diagrams corresponding to Eq. (5.5) are built out of just particle lines, tachyon propagators (which we formerly called noise propagators) and vertices where one end of a tachyon propagator meets two particle lines. To (lowest) order $\gamma$, the nonvanishing diagrams, which are responsible for collapse and which replace those in Figs. 3a,b,c, are shown in Figs. 4a,b,c. We shall obtain an expression for their contribution here. However, in order to bring out the similarity to nonrelativistic CSL, we shall not use diagrammatic methods.

The density matrix (5.5) to lowest order is

$$
\begin{aligned}
& \rho(T / 2)=\rho(-T / 2)-\frac{1}{2} \gamma(2 M)^{2} \int_{-\frac{T}{2}}^{\frac{T}{2}} d x d x^{\prime} G\left(x-x^{\prime}\right) \\
& {\left[\begin{array}{r}
\mathcal{T}: \psi^{2}(x):: \psi^{2}\left(x^{\prime}\right): \rho(-T / 2)+\rho(-T / 2) \mathcal{T}_{\mathcal{R}}: \psi^{2}(x):: \psi^{2}\left(x^{\prime}\right): \\
\left.\quad-2: \psi^{2}(x): \rho(-T / 2): \psi^{2}\left(x^{\prime}\right):\right]
\end{array}\right.}
\end{aligned}
$$

$\left(\mathcal{T}_{\mathcal{R}}\right.$ is the time-reversing operator $)$. Utilizing

$$
\mathcal{T}: \psi^{2}(x):: \psi^{2}\left(x^{\prime}\right):=: \psi^{2}\left(x^{\prime}\right):: \psi^{2}(x):+\Theta\left(t-t^{\prime}\right)\left[\psi^{2}(x), \psi^{2}\left(x^{\prime}\right)\right]
$$

and taking advantage of the $x \leftrightarrow x^{\prime}$ symmetry allows writing Eq. (6.1) as

$$
\begin{aligned}
\rho(T / 2)= & \rho(-T / 2)-\frac{1}{2} \gamma(2 M)^{2} \int_{-\frac{T}{2}}^{\frac{T}{2}} d x d x^{\prime} G\left(x-x^{\prime}\right)\left[: \psi^{2}(x):\left[: \psi^{2}\left(x^{\prime}\right):, \rho(-T / 2)\right]\right] \\
& +\frac{i}{4} \gamma(2 M)^{2} \int_{-\frac{T}{2}}^{\frac{T}{2}} d x d x^{\prime} G\left(x-x^{\prime}\right) \epsilon\left(t-t^{\prime}\right)\left[i\left[\psi^{2}(x), \psi^{2}\left(x^{\prime}\right)\right], \rho(-T / 2)\right]
\end{aligned}
$$

Now we note the very important relation

$$
\int_{-\frac{T}{2}}^{\frac{T}{2}} d x^{\prime} G\left(x-x^{\prime}\right) \psi_{ \pm}^{2}\left(x^{\prime}\right) \longrightarrow 0 \quad \text { as } T \rightarrow \infty
$$

where $\psi_{+}\left(x^{\prime}\right)$ and $\psi_{-}\left(x^{\prime}\right)$ are the positive and negative frequency parts respectively of $\psi\left(x^{\prime}\right)$. The expression (6.4) vanishes because, in momentum space, the integrand is $\sim \delta\left(k^{2}+\mu^{2}\right) \delta^{4}\left[k \pm\left(p_{1}+p_{2}\right)\right]$, where $p_{1}$ and $p_{2}$ are timelike, and the sum of two timelike four-momenta cannot equal a spacelike four-momentum. Eq. (6.4) guarantees that no pair production out of the vacuum arises from the first perturbation term in Eq. (6.3), as it permits : $\psi^{2}(x)$ : to be replaced by $2 \psi_{-}(x) \psi_{+}(x)$. Eq. (6.4) will turn out to be crucial in obtaining no particle creation out of the vacuum to any order in the RCSL model discussed in the next section, and crucial for eliminating the need for renormalization.

There is no pair production out of the vacuum arising from the second perturbation term in Eq. (6.3) either, since its pair production/annihilation part has the form

$$
\int_{-\frac{T}{2}}^{\frac{T}{2}} d x d x^{\prime} \psi_{ \pm}(x) \psi_{ \pm}\left(x^{\prime}\right) F\left(x-x^{\prime}\right) \longrightarrow 0 \quad \text { as } T \rightarrow \infty
$$

(because in momentum space the integrand is $\sim \delta^{4}\left(p+p^{\prime}\right)=0$ ). Thus we finally obtain, for large $T$, 


$$
\begin{aligned}
& \rho(T / 2)=\rho(-T / 2)-2 \gamma(2 M)^{2} \int_{-\frac{T}{2}}^{\frac{T}{2}} d x d x^{\prime} G\left(x-x^{\prime}\right)\left[\psi_{-}(x) \psi_{+}(x),\left[\psi_{-}\left(x^{\prime}\right) \psi_{+}\left(x^{\prime}\right), \rho(-T / 2)\right]\right] \\
& +i 2 \gamma(2 M)^{2} \int_{-\frac{T}{2}}^{\frac{T}{2}} d x d x^{\prime} G\left(x-x^{\prime}\right) \epsilon\left(t-t^{\prime}\right)<0\left|i\left[\psi(x), \psi\left(x^{\prime}\right)\right]\right| 0>\left[\psi_{-}(x) \psi_{+}\left(x^{\prime}\right), \rho(-T / 2)\right]
\end{aligned}
$$

The first perturbation term in Eq. (6.5) describes collapse. (It corresponds to diagrams $4 \mathrm{~b}, \mathrm{c}$ as well as the real part of diagrams 4a.) Some of its consequences will be obtained in section IX. It is quite similar in form to the comparable nonrelativistic CSL expression (obtained from the perturbation expansion of Eq. (2.7)):

$$
\begin{aligned}
\rho(T / 2)=\rho(-T / 2)-\frac{\lambda}{2} \int_{-\frac{T}{2}}^{\frac{T}{2}} d x d x^{\prime} \delta\left(t-t^{\prime}\right) e^{-\frac{1}{4 a^{2}}\left(\mathbf{x}-\mathbf{x}^{\prime}\right)^{2}} \\
\cdot\left[\xi^{\dagger}(\mathbf{x}, t) \xi(\mathbf{x}, t),\left[\xi^{\dagger}\left(\mathbf{x}^{\prime}, t\right) \xi\left(\mathbf{x}^{\prime}, t\right), \rho(-T / 2)\right]\right]
\end{aligned}
$$

The second perturbation term in Eq. (6.5) is a unitary (noncollapse) evolution. This term comes from the imaginary part of the diagrams 4a. It is the divergent, imaginary, part of the self-energy diagrams 4a. While it may be removed by mass renormalization, we shall remove it a different way.

\section{RCSL}

The $\mathrm{RCSL}_{2}$ choice of tachyonic $\tilde{G}\left(k^{2}\right)$ means there is no particle production out of the vacuum in order $\gamma$. However, there is pair production out of the vacuum in order $\gamma^{2}$ via the diagram in Fig. 5. The culprit is the off-shell fourmomentum (i.e., $p^{\nu}$ can take on any value) of the Feynman propagator. If the propagator were on-shell (i.e., $p^{2}=M^{2}$ ), energy conservation at the lower vertices in Fig. 5 would make the contribution of the diagram vanish for the same reason that (6.4) vanishes.

We shall now see how to achieve this so as to eliminate vacuum excitation to this order, and indeed to all orders. This can be accomplished if we focus on an apparently unrelated task, eliminating the second perturbation term in Eq. (6.5). It is not unrelated however, because this term depends upon $\epsilon\left(t-t^{\prime}\right)<0\left|i\left[\psi(x), \psi\left(x^{\prime}\right)\right]\right| 0>$ whose Fourier transform is $\mathcal{P}\left(p^{2}-m^{2}\right)^{-1}$, the off-shell (imaginary) part of the Feynman propagator.

Consider the statevector and density matrix evolution equations (3.4), (3.5) where we replace $F(x)=2 M: \psi^{2}(x)$ : by $F(x)=2 M: \psi^{2}(x):+F_{1}(x)$ with

$$
F_{1}(x) \equiv \gamma(2 M)^{2} \int_{T_{0}}^{t} d x^{\prime} \eta\left(x^{\prime}\right) i\left[\psi^{2}(x), \psi^{2}\left(x^{\prime}\right)\right]
$$

(it is to be understood that the time-ordering operation is to treat the operator $F_{1}(x)$ as a function of $t$ ). When this new expression for $F(x)$ is put into the density matrix (3.5), $F_{1}(x)$ adds to the lowest order expression (6.5) a contribution which is the negative of the last term in (6.5).

What has happened is this: where $\mathcal{T}: \psi^{2}(x):: \psi^{2}\left(x^{\prime}\right):$ appears in the lowest order Feynman diagrams, $F_{1}(x)$ adds $-\Theta\left(t-t^{\prime}\right)\left[\psi^{2}(x), \psi^{2}\left(x^{\prime}\right)\right]$ to it. Thus, as can be seen from Eq. (6.2), the extra term $F_{1}(x)$ effectively removes the time-ordering operation.

It also turns out (we shall spare the reader the details) that use of (7.1) also removes the $\mathcal{T}$ operator in the next order, from the expression for the pair production diagram of Fig. 5. But, because of Eq. (6.4),

$$
\int d x G\left(x-x^{\prime}\right): \psi^{2}(x):=\int d x G\left(x-x^{\prime}\right) 2 \psi_{-}(x) \psi_{+}(x)
$$

replacing $\mathcal{T}: \psi^{2}(x):: \psi^{2}\left(x^{\prime}\right)$ : by $: \psi^{2}(x):: \psi^{2}\left(x^{\prime}\right)$ : has the effect, for the diagram of Fig. (5), of replacing the term $\mathcal{T}: \psi^{2}(x):: \psi^{2}\left(x^{\prime}\right):$, which can create particle pairs out of the vacuum, by the term $4 \psi_{-}(x) \psi_{+}(x) \psi_{-}\left(x^{\prime}\right) \psi_{+}\left(x^{\prime}\right)$ which annihilates the vacuum. Adding $F_{1}(x)$ has made the contribution of the diagram of Fig. 5 vanish: there is now no vacuum excitation to order $\gamma^{2}$.

It is now clear that a way to remove vacuum excitation to all orders is to keep the $\mathrm{RCSL}_{2}$ statevector evolution except remove the time ordering operator $\mathcal{T}$ :

$$
\left|\psi, \sigma>=\int D \eta e^{-\gamma \int_{-\infty}^{\infty} d x d x^{\prime} \eta(x) G^{-1}\left(x-x^{\prime}\right) \eta\left(x^{\prime}\right)} e^{i \int_{\sigma_{0}}^{\sigma} d x \eta(x)\left[w(x)-2 \gamma(2 M): \psi^{2}(x):\right]}\right| \psi, \sigma_{0}>
$$


This model I shall call RCSL.

When the time ordering operator $\mathcal{T}$ is removed, the form (3.1) and its Fourier transform form (3.4) are generally no longer equal. The Fourier transform form, (7.3), has to be chosen to define RCSL because only then is the integrated probability density (2.2) equal to 1 . The RCSL density matrix is (5.5) without the $\mathcal{T}$-operation:

$$
\rho(\sigma)=\int D \eta e^{-2 \gamma \int_{-\infty}^{\infty} d x d x^{\prime} \eta(x) G^{-1}\left(x-x^{\prime}\right) \eta\left(x^{\prime}\right)} e^{-i 2 \gamma(2 M) \int_{\sigma_{0}}^{\sigma} d x \eta(x)\left[: \psi^{2}(x): \otimes 1-1 \otimes: \psi^{2}(x):\right]} \rho\left(\sigma_{0}\right)
$$

It is easily seen that the trace of (7.4) (the integrated probability density) is 1 .

When (7.4) is expanded in a perturbation series and (6.4) is employed, the result for large $T$ is

$$
\begin{gathered}
\rho(T / 2)=\sum_{n=0}^{\infty} \frac{\left(-16 \gamma M^{2}\right)^{n}}{(2 n) !} \int_{-T / 2}^{T / 2} d x_{1} \ldots d x_{2 n} \sum_{C} G\left(x_{i_{1}}-x_{i_{2}}\right) \ldots G\left(x_{i_{2 n-1}}-x_{i_{2 n}}\right) \\
\cdot\left[\psi_{-}\left(x_{1}\right) \psi_{+}\left(x_{1}\right),\left[\ldots\left[\psi_{-}\left(x_{2 n}\right) \psi_{+}\left(x_{2 n}\right), \rho(-T / 2)\right] \ldots\right]\right]
\end{gathered}
$$

(the summation is over the $(2 n-1)$ !! pairing combinations of the integers $1, \ldots 2 \mathrm{n}$ ). Thus, two features, choice of tachyonic $\tilde{G}\left(k^{2}\right)$ and removal of the time-ordering operation, has resulted in a model which is free from vacuum excitation, dependent as it is on operators only in the combination $\psi_{-}(x) \psi_{+}(x)$.

Indeed, it is completely finite: there is no need for renormalization.

RCSL is Lorentz invariant: the arguments in Appendix C may just as well have been applied there to the Fourier transform representation of the statevector (the only additional information needed is that $\eta(x)$ transforms as a Lorentz scalar). With or without the $\mathcal{T}$ - operation, operators and variables transform as stated, so the demonstration goes through as before.

Incidentally, it may be remarked that one would not have a Lorentz invariant statevector or density matrix if such products as $\mathcal{T} \psi_{-}(x) \psi_{+}\left(x^{\prime}\right)$ appears in them (the time-ordering is not Lorentz invariant since $\psi_{-}(x)$ and $\psi_{+}\left(x^{\prime}\right)$ do not commute for spacelike $\left.x-x^{\prime}\right)$ but $\psi_{-}(x) \psi_{+}\left(x^{\prime}\right)$ does appear in RCSL expressions and it is Lorentz invariant. Indeed, one could replace : $\psi^{2}(x)$ : in Eqs. (7.3), (7.4) by $2 \psi_{-}(x) \psi_{+}(x)$ ) without affecting the S-matrix behavior, but also without violating Lorentz invariance. So far I have no reason for choosing one form over the other.

It is also worth remarking that removing the time-ordering operation in a normal field theory with an interaction of the form $\psi^{2}(x) \phi(x)$ is trivial (if the mass of the $\phi$ particle is less than twice the mass of the $\psi$ ) since, if all the particles are on-shell, energy-momentum conservation at each vertex forbids any evolution. It is only with the $\phi$ particle replaced by a tachyon that evolution is possible.

RCSL describes collapse for an individual statevector. For the usual test, we set $H=0$ in Eq. (7.3) so that the unitary evolution does not interfere with the collapse evolution. When we replace $\psi_{-}(x) \psi_{+}(x)$ by $\psi_{-}(\mathbf{x}, \mathbf{0}) \psi_{+}(\mathbf{x}, \mathbf{0})$, then all operators commute. It is then irrelevant whether or not a time-ordering operator is present and the demonstration of individual collapse is that in Appendix A.

One may represent the terms in the expansion (7.5) by Feynman diagrams. The usual off-shell Feynman propagator for the particle, $<0\left|\mathcal{T} \psi(x) \psi\left(x^{\prime}\right)\right| 0>$, is replaced by the on-shell positive-energy propagator $<0\left|\psi(x) \psi\left(x^{\prime}\right)\right| 0>$. Since the tachyon propagator is on-shell as well, we may think of all diagrams as representing real (as opposed to virtual) particles emitting or absorbing real tachyons. It is easy to calculate from conservation of energy-momentum that a particle of mass $M$ at rest emits a negative energy tachyon of total energy $-\mu^{2} / 2 M$, and goes off with kinetic energy $\mu^{2} / 2 M$. In a sense, the GRW spontaneous "hits" in CSL may be thought of as replaced by the spontaneous emission/absorption of tachyons in RCSL as the cause of collapse.

\section{LOCALITY AND NONLOCALITY IN RCSL}

We have removed the time-ordering operation as one way to obtain a finite theory. One expects that it will lead to nonlocal behavior. In this section we explore what that behavior is and how bad it is.

\section{A. No Time-Ordering}

In Appendix $\mathbb{E} 1$ it is shown how to write $\exp -i \int d t V(t)$ in the form $\mathcal{T}\left\{\exp -i \int d t H_{\mathrm{eff}^{(}}(t)\right\}$. As pointed out there, $V$ and $H_{\text {eff }}$ are identical to order $\gamma$, but to order $\gamma^{2}$ and higher, $H_{\text {eff }}$ has a nonlocal part which transports a particle from $x$ to $x^{\prime} . x$ and $x^{\prime}$ are timelike related, but the transport can be in the backwards as well as the forwards time direction. Higher order terms describe a sequence of backwards and forwards motions in time between $x$ and $x^{\prime}$. 
Another way to see that backwards-in-time motion is characteristic of no time-ordering is as follows. The standard evolution of a statevector in the interaction picture is

$$
\begin{aligned}
& \mathcal{T}\left\{e^{-i \int_{0}^{T} d t V(t)}\right\} \mid L_{0}, R_{0}>= \sum_{n}(-i)^{n} e^{i H_{0} T} \int_{0}^{T} d t_{n} \cdots \int_{0}^{t_{3}} d t_{2} \int_{0}^{t_{2}} d t_{1} \cdot \\
& e^{-i H_{0}\left(T-t_{n}\right)} V(0) \cdots e^{-i H_{0}\left(t_{3}-t_{2}\right)} V(0) e^{-i H_{0}\left(t_{2}-t_{1}\right)} V(0) e^{-i H_{0} t_{1}} \mid L_{0}, R_{0}>
\end{aligned}
$$

In (8.1), particles evolve freely for $t_{1}$ sec, change momenta, evolve freely for $\left(t_{2}-t_{1}\right)$ sec, change momenta, etc., until time $T$. All the motion is forward in time. (The $\exp i H_{0} T$ at the left end of the integrals is an artifact of the interaction picture, not a true backwards-in-time motion, and is removed in the Schrodinger picture.) Now consider this evolution without the time-ordering operation:

$$
\begin{aligned}
& e^{-i \int_{0}^{T} d t V(t)} \mid L_{0}, R_{0}>=\sum_{n} \frac{(-i)^{n}}{n !} e^{i H_{0} T} \int_{0}^{T} d t_{n} e^{-i H_{0}\left(T-t_{n}\right)} V(0) e^{-i H_{0} t_{n}} \ldots \\
& e^{i H_{0} T} \int_{0}^{T} d t_{2} e^{-i H_{0}\left(T-t_{2}\right)} V(0) e^{-i H_{0} t_{2}} e^{i H_{0} T} \int_{0}^{T} d t_{1} e^{-i H_{0}\left(T-t_{1}\right)} V(0) e^{-i H_{0} t_{1}} \mid L_{0}, R_{0}>
\end{aligned}
$$

In (8.2), particles evolve freely for $t_{1}$ sec, change momenta, evolve freely for $\left(T-t_{1}\right)$ sec, and then evolve freely backwards in time for $T$ sec, and then do it all over again repeatedly.

Yet a third way to see this is to write (8.2) in a different way, e.g., the second order term may be written as

$$
\begin{gathered}
\int_{0}^{T} d t_{2} V\left(t_{2}\right) \int_{0}^{T} d t_{1} V\left(t_{1}\right)=e^{i H_{0} T}\left[\int_{0}^{T} d t_{2} e^{-i H_{0}\left(T-t_{2}\right)} V(0) \int_{0}^{t_{2}} d t_{1} e^{-i H_{0}\left(t_{2}-t_{1}\right)} V(0) e^{-i H_{0} t_{1}}\right. \\
\left.+\int_{0}^{T} d t_{2} e^{-i H_{0}\left(T-t_{2}\right)} V(0) \int_{t_{2}}^{T} d t_{1} e^{i H_{0}\left(t_{2}-t_{1}\right)} V(0) e^{-i H_{0} t_{1}}\right]
\end{gathered}
$$

as the sum of a time-ordered evolution, and of an evolution which goes freely backwards in time between the two interactions.

A sequence of backward and forward motions in time can lead to spacelike (superluminal) transport, but it doesn't have to.

\section{B. Particle Motion In RCSL}

In this subsection we shall see, for particles of mass $M>>\mu$, spacelike transport only occurs at a very high order of perturbation theory, and so has a negligibly small probability.

The motion of a particle can be envisioned as a sequence of emissions/absorptions of tachyons. In Appendix E2, the evolution over time $T$ of the wavefunction of a particle initially localized in position and momentum is calculated to lowest order (diagram 4c). For example, if the particle is initially at rest (i.e., described by a wavepacket centered around momentum 0 and, say, around the position $\mathbf{x} \approx 0$ ), its wavepacket spreads a distance $(\mu / M) c T$ in the direction of the momentum $\mathbf{p}=\mu c \hat{\mathbf{p}}$ which it gains from an emitted tachyon. In addition to this spread, the resulting wavefunction acquires the momentum $\mathbf{p}$ (acquires a phase factor $\exp i \mathbf{p} \cdot \mathbf{x}$ ).

This is precisely the same as the behavior of a collection of classical particles, each of which starts at $\mathbf{x}=0$ at $t=0$ but, at a random time $t$ in the interval $(0, T)$, receives an impulse resulting in momentum $\mathbf{p}$ : a particle receiving the impulse at $t=0$ travels the farthest distance, $(\mu / M) c T$.

The backwards in time evolution $\exp i H_{0} T$ then carries the wavefunction a distance $(\mu / M) c T$ in the opposite direction from $\hat{\mathbf{p}}$ so that an elongated wavefunction stretching from $\approx-(\mu / M) c T \hat{\mathbf{p}}$ to the origin and describing a particle with momentum $\mathbf{p}$, awaits the next evolution. From the point of view of the classical particle picture, the particle which received its impulse at $t=0$ ends up where it started, at $\mathbf{x}=0$, while the particle which received its impulse at $t=T$ ends up at $-(\mu / M) c T \hat{\mathbf{p}}$.

The tachyons are, however, emitted in random directions so the complete wavefunction arising from diagram $4 \mathrm{c}$ is spread over a sphere of radius $(\mu / M) c T$. Thus, at each vertex the wavefunction spread is less than the width $c T$ of the lightcone. One might think that the lightcone width would be exceeded if the particle initally moves with speed near $c$ so it is initially close to the lightcone, but that is not so: at relativistic speeds the spread is Lorentz contracted, and the wavefunction remains within the lightcone. 
Now consider a higher order diagram, e.g., the iteration of fig 4c, a so-called "ladder diagram" (other diagrams of the same order with more complicated configurations engender no more spread than this one). At each vertex there is a further spread. One might think that the situation of largest spread occurs if each subsequent evolution described by the succeeding integrals in (8.2) involves a tachyon emitted/absorbed in the same direction so that the particle is accelerated in the same direction in each order. However, as shown in Appendix E2, each subsequent spread is slightly less than the previous one (precisely the same as the Lorentz transformed shrinking of the spread due to increased velocity of the particle) and the net spread after an arbitrary number of such interactions is always within the light cone.

However, there are scenarios for a sequence of tachyon interactions which result in a spread outside the lightcone. Here is the one that spreads the most. It can be understood from the classical particle picture. Consider two successive evolutions of a particle initially at rest. In the first evolution, the particle starts at rest at the origin, and gets its momentum $\mathbf{p}$ at the latest possible time, $t=T$, so that after travelling backward-in-time for time $T$ it is located at $-(\mu / M) c T \hat{p}$. In the second evolution, the particle gets the opposite momentum $-\mathbf{p}$ at the earliest possible time, $t=0$. Thus, the particle comes to rest at location $-(\mu / M) c T \hat{p}$, and stays there for the rest of that evolution. The net effect of these two evolutions is that the particle ends up at rest at $-(\mu / M) c T \hat{p}$, and if each successive pair of evolutions is the same, after more than $2 M / \mu$ evolutions the particle wavefunction is outside the lightcone. But, this is a very improbable. The first reason is that the phase space volume in which the succession of tachyons put their momentum in the right directions is small. The second reason is that it occurs at a very high order of perturbation theory $\left(\approx 10^{6}\right.$ for an electron, $\left(\approx 10^{9}\right.$ for a nucleon $)$. The wavefunction arising from the set of ladder diagrams is essentially that of a random walk of spread $\approx(\mu / M) c T$ (faster than the usual random walk $\sim \sqrt{T}$ ), wuth a very small tail outside the lightcone $c T$.

To summarize, in the time-ordered situation, particle wavefunctions rigorously stay within their lightcones: in the no time-ordered situation this is not so, but it is true to a very high probability. This is what we need in the next subsection to show that the model is local to a very high probability.

\section{Locality and RCSL}

First let's review the locality criteria as manifested in a time-ordered evolution.

Consider separated spatial regions $R_{0}$ and $L_{0}$ and a (possibly entangled) initial state $\left|L_{0}, R_{0}\right\rangle$ with some particles in $R_{0}$ and the rest in $L_{0}$. Call $R$ and $L$ the spacetime four-volume bounded by the lightcones emanating respectively from $R_{0}$ and $L_{0}$ over the time interval $T$. Call $R_{t}$ and $L_{t}$ the intersections of $R$ and $L$ with the spacelike hyperplane $t$ for $0 \leq t \leq T$. Suppose that $R_{T}$ and $L_{T}$ do not overlap. The standard evolution of a statevector in the interaction picture is

$$
\begin{aligned}
& \mathcal{T}\left\{e^{-i \int_{0}^{T} d t V(t)}\right\} \mid L_{0}, R_{0}>= \\
& \sum_{n}(-i)^{n} e^{i H_{0} T} \int_{0}^{T} d t_{n} \cdots \int_{0}^{t_{3}} d t_{2} \int_{0}^{t_{2}} d t_{1} \\
& \quad e^{-i H_{0}\left(T-t_{n}\right)} V(0) \cdots e^{-i H_{0}\left(t_{3}-t_{2}\right)} V(0) e^{-i H_{0}\left(t_{2}-t_{1}\right)} V(0) e^{-i H_{0} t_{1}} \mid L_{0}, R_{0}>
\end{aligned}
$$

It often occurs that $V(t)\left|L, R>=\left[V_{L}(t)+V_{R}(t)\right]\right| L, R>$, where $\mid L, R>$ is any state with particles only residing within $R$ and $L$ and $V_{L}(t), V_{R}(t)$ act only on particles within their respective lightcones. This requires that the free evolution $\exp -i H_{0} t$ moves particles within their lightcones. This is automatic in a local relativistic quantum field theory. In nonrelativistic quantum mechanics this needs an appropriately chosen initial wavefunction (e.g., wavepacket width $>>M^{-1}$ ). It also requires $V(0)$ to give no interaction between particles in $R$ and particles in $L$. This is automatic in a local relativistic quantum field theory. In nonrelativistic quantum mechanics this is satisfied for a potential that is shorter range than the minimum separation between $R_{T}$ and $L_{T}$.

If this is the case, then taking the trace over particle states in $R_{T}$ results in

$$
\begin{aligned}
\operatorname{Tr}_{R}\{\rho(T)\}= & \operatorname{Tr}_{R}\left\{\mathcal{T}\left[e^{-i \int_{0}^{T} d t V_{R}(t)}\right] \mathcal{T}\left[e^{-i \int_{0}^{T} d t V_{L}(t)}\right] \mid L_{0}, R_{0}>\right. \\
& \left.\cdot<L_{0}, R_{0} \mid \mathcal{T}_{\mathcal{R}}\left[e^{i \int_{0}^{T} d t V_{L}(t)}\right] \mathcal{T}_{\mathcal{R}}\left[e^{i \int_{0}^{T} d t V_{R}(t)}\right]\right\} \\
= & \mathcal{T}\left[e^{-i \int_{0}^{T} d t V_{L}(t)}\right] \operatorname{Tr}_{R}\left\{\left|L_{0}, R_{0}><L_{0}, R_{0}\right|\right\} \mathcal{T}_{\mathcal{R}}\left[e^{i \int_{0}^{T} d t V_{L}(t)}\right]
\end{aligned}
$$

This is the hallmark of locality: that no evolution in $R$ has any effect on what happens in $L$. In particular, in an interacting theory, this means that no manipulation by an apparatus in $R$ during the time interval $(0, T)$ has any effect 
on $L$. In a noninteracting theory like the present one this interpretation is not useful (apparatuses require interaction) but the requirement $8.4 \mathrm{~b}$ ) stands. For example, if the initial statevector is the direct product $\left|L_{0}>\right| R_{0}>$, it means that the evolution in $L$ is independent of whatever state is initially in $R$.

Now consider the non time-ordered evolution. In the the previous subsection we have shown that a particle initially at the origin, although it may spread in each successive perturbation order, stays within the cylindrical four-volume of spatial radius $c T$ and time interval $0 \leq t \leq T$ with a probability that is very close to 1 . Suppose this were true with probability 1 . Then the effect of $N(x)=\psi_{-}(x) \psi_{+}(x)$ in the perturbation series of (7.5) could be written as

$$
\begin{aligned}
\rho(T)=\sum_{n=0}^{\infty} \frac{\left(-16 \gamma M^{2}\right)^{n}}{(2 n) !} \int_{T_{0}}^{T} d x_{1} \ldots d x_{2 n} \sum_{C} G\left(x_{i_{1}}-x_{i_{2}}\right) \ldots G\left(x_{i_{2 n-1}}-x_{i_{2 n}}\right) \\
\cdot\left[N_{L}\left(x_{1}\right)+N_{R}\left(x_{1}\right),\left[\ldots\left[N_{L}\left(x_{2 n}\right)+N_{R}\left(x_{2 n}\right),\left|L_{0}, R_{0}><L_{0}, R_{0}\right|\right] \ldots\right]\right]
\end{aligned}
$$

where $N_{L}(\mathbf{x}, t)$ has $(\mathbf{x}, t)$ lying in the cylindrical four-volume surrounding the $L$-particle (forwards and backwards-intime) world-lines, i.e., a region of spatial extension $L_{T}$ at each time $0 \leq t \leq T$, and similarly for $N_{R}(\mathbf{x}, t)$. Call these cylindrical volumes $L^{\prime}$ and $R^{\prime}$. $N_{L}(\mathbf{x}, t)$ would only act on particles in $L^{\prime}$ and the result of the action would leave the particle in $L^{\prime}$, and similarly for $N_{R}(\mathbf{x}, t)$. Thus, although $N_{L}(x)$ and $N_{R}\left(x^{\prime}\right)$ do not commute for all $x, x^{\prime}$ nonetheless, in the integrand of (8.5), one may utilize

$$
\begin{gathered}
{\left[N_{L}\left(x_{i}\right)+N_{R}\left(x_{i}\right),\left[N_{L}\left(x_{j}\right)+N_{R}\left(x_{j}\right), . .\right]\right]=\left[N_{L}\left(x_{i}\right),\left[N_{L}\left(x_{j}\right), . .\right]\right]+\left[N_{R}\left(x_{i}\right),\left[N_{L}\left(x_{j}\right), . .\right]\right]} \\
+\left[N_{R}\left(x_{j}\right),\left[N_{L}\left(x_{i}\right), . .\right]\right]+\left[N_{R}\left(x_{i}\right),\left[N_{R}\left(x_{j}\right), . .\right]\right]
\end{gathered}
$$

to put all $N_{R}$ commutators to the outside of the $N_{L}$ commutators. After relabelling of indices and summing of the series one obtains

$$
\begin{aligned}
& \rho(T)=\int D \eta e^{-2 \gamma \int_{-\infty}^{\infty} d x d x^{\prime} \eta(x) G^{-1}\left(x-x^{\prime}\right) \eta\left(x^{\prime}\right)} e^{-i 2 \gamma(2 M) \int_{T_{0}}^{T} d x \eta(x)\left[N_{R}(x) \otimes 1-1 \otimes N_{R}(x)\right]} \\
& \cdot e^{-i 2 \gamma(2 M) \int_{T_{0}}^{T} d x \eta(x)\left[N_{L}(x) \otimes 1-1 \otimes N_{L}(x)\right]}\left|L_{0}, R_{0}><L_{0}, R_{0}\right|
\end{aligned}
$$

It immediately follows from $(8.6)$ that

$$
\begin{aligned}
\operatorname{Tr}_{R}\{\rho(T)\} & =\int D \eta e^{-2 \gamma \int_{-\infty}^{\infty} d x d x^{\prime} \eta(x) G^{-1}\left(x-x^{\prime}\right) \eta\left(x^{\prime}\right)} \\
& \cdot e^{-i 2 \gamma(2 M) \int_{T_{0}}^{T} d x \eta(x)\left[N_{L}(x) \otimes 1-1 \otimes N_{L}(x)\right]} \operatorname{Tr}_{R}\left\{\left|L_{0}, R_{0}><L_{0}, R_{0}\right|\right\}
\end{aligned}
$$

which, as in Eq. (8.4), is the hallmark of locality.

While Eq. (8.7) is not strictly true in RCSL, the terms by which it deviates are of very small magnitude, so we say that RCSL is local to a high degree of accuracy.

It is worth remarking that if all particles are distinguishable (so there are as many quantum fields as particles, and $N(x)$ is replaced by the sum $\sum_{k} N_{k}(x)$ in all expressions) then (8.7) is exact if one traces over the identifiable particles which start in $R$, no matter where they evolve to. At the ensemble level, this is basically a theory of particles which do not interact among themselves (the $N_{k}(x)$ all commute) in spite of the tachyonic interaction at the individual level.

It is perhaps also worth remarking that, with a local theory, there can be no causal loops (e.g., no sending messages to kill one's father before one is born), even though there are tachyonic interactions. The essential reason is that the tachyons are emitted spontaneously: one cannot send a message when one does not have control of the messenger. This is the same reason why one cannot communicate superluminally by making one part of an entangled separated system collapse: one cannot force a particular outcome of the collapse because one does not have control of the tachyonic noise field.

\section{SOME RELATIVISTIC RESULTS}

Now that we have a finite relativistic collapse model, we may use it. To illustrate, we shall present two calculations to order $\gamma$, using (7.5):

$$
\rho(T / 2)=\rho(-T / 2)-2 \gamma(2 M)^{2} \int_{-\frac{T}{2}}^{\frac{T}{2}} d x d x^{\prime} G\left(x-x^{\prime}\right)\left[\psi_{-}(x) \psi_{+}(x),\left[\psi_{-}\left(x^{\prime}\right) \psi_{+}\left(x^{\prime}\right), \rho(-T / 2)\right]\right]
$$


First we consider the energy increase of $n$ free particles due to collapse. In Appendix 国 we calculate $\bar{H}(T / 2) \equiv$ $\operatorname{Tr} H \rho(T / 2)$, with the result

$$
\bar{H}(T / 2)=\bar{H}(-T / 2)+\frac{1}{2 \pi^{2}} n \gamma T \frac{\mu^{3}}{M} \sqrt{1+\left(\frac{\mu}{2 M}\right)^{2}}
$$

This may be compared with the CSL energy increase which follows from Eq. (2.7):

$$
\bar{H}(T / 2)=\bar{H}(-T / 2)+\frac{3}{4} n \lambda T \frac{\mu^{2}}{M}
$$

(using $a^{-1}=\mu$ ). They are identical (for $M>>\mu$, apart from numerical factors) if we identify $\gamma=\lambda \mu^{-1}: \gamma \approx 10^{-32}$, with GRW's choice of parameter values. How could this small a dimensionless number be accounted for? We note, following Diosi [16] that $G M_{N}^{2} \approx 10^{-38}$ with $M_{N}=$ proton or neutron mass. We may set

$$
\gamma=\kappa G M_{N}^{2}
$$

with $\kappa \approx 10^{6}$ for the GRW choice, while Diosi has suggested $\kappa \approx 1$.

The nonrelativistic energy increase (9.3) may be thought of as arising from the GRW "hitting" process. This narrowing of a particle's wavefunction to distance $\approx \mu^{-1}$ on the time scale $\lambda^{-1}$ imparts to the particle a momentum $\sim \mu$ and thus an energy $\sim \mu^{2} / 2 M$. Likewise, the relativistic energy increase (9.2) may be thought of as the consequence of a free particle suddenly emitting a tachyon, as represented in the diagram (4c). A particle at rest, which emits a negative energy tachyon, gains energy $\mu^{2} / 2 M$.

According to Eq. (9.2), if the collapse works on particles of any mass, the mean energy imparted to zero mass particles becomes infinite $\sim M^{-2}$. However, experiments [8,13,14 indicate that particles should be coupled to $w(\mathbf{x}, t)$ via their mass. To take account of different types of particles, in (7.3), (7.4) one should effect the replacement

$$
\gamma(2 M): \psi^{2}(x): \longrightarrow \gamma \sum_{i} \frac{M_{i}}{M_{N}}\left(2 M_{i}\right): \psi_{i}^{2}(x):
$$

$\left(\psi_{i}(x)\right.$ is the field of the particle with mass $\left.M_{i}\right)$. Then Eq. (9.2) becomes, for the ith particle type,

$$
\bar{H}(T / 2)-\bar{H}(-T / 2)=\frac{1}{2 \pi^{2}} n \gamma T \frac{\mu^{3} M_{i}}{M_{N}^{2}} \sqrt{1+\left(\frac{\mu}{2 M_{i}}\right)^{2}} \longrightarrow \frac{1}{(2 \pi)^{2}} n \gamma T \frac{\mu^{4}}{M_{N}^{2}}
$$

in the limit $M_{i} \longrightarrow 0$. This zero-mass rate is $\approx\left(\mu / M_{N}\right) \approx 10^{-9}$ times as big as the rate (9.2) for a proton. Thus, even if (9.6) were to apply to photons or (zero mass) neutrinos, of which the universe contains $\approx 10^{8}$ times as many as protons, the energy increase in the universe due to collapse of these particles would be comparable to the energy increase in the universe due to the protons alone. However, in the strict zero mass limit, with use of (9.5), the probability/sec of the tachyon reaction vanishes. Such a never-occurring process, even one which produces an infinite energy (so the average is (9.6)) has little physical significance.

Now we turn to consideration of the collapse rate for a single particle in the state $\alpha|L>+\beta| R>$, where $\mid L>$ and $\mid R>$ are reasonably well-localized states separated by a great distance $>>c T$, but otherwise identical. The off-diagonal matrix element $<L|\rho(T / 2)| R>$ is calculated in Appendix G. The result G6, applied to the ith type of particle, is

$$
<L|\rho(T / 2)| R>=\alpha \beta^{*}\left[1-\frac{2}{\pi^{2}} \gamma T \frac{\mu M_{i}^{3}}{M_{N}^{2}} \sqrt{1+\left(\frac{\mu}{2 M_{i}}\right)^{2}} \int \frac{d \mathbf{p}}{2 E}|\Psi(\mathbf{p})|^{2}\right]
$$

where $\Psi(\mathbf{p})$ is the particle's normalized wavefunction in momentum space. (9.7) arises from the "self-energy" diagrams 4a.) The comparable CSL result, obtainable from (2.7), is:

$$
<L|\rho(T / 2)| R>=\alpha \beta^{*}\left[1-\lambda T \frac{M_{i}^{2}}{M_{N}^{2}}\right]
$$

They are identical (for $M_{i}>>\mu$, apart from numerical factors) when $\gamma=\lambda \mu^{-1}$, provided $|\Psi(\mathbf{p})|^{2}$ only has support for nonrelativistic momenta: then we may set $E \approx M_{i}$, and take it out of the integral in (9.7) (the remaining integral=1).

In the relativistic domain, the most interesting feature of $(9.7)$ is the dependence of the integrand on $1 / E$. If $|\Psi(\mathbf{p})|^{2}$ is fairly well localized in momentum space around $\mathbf{p}_{0}$, we may set $E \approx E_{0}$ and take it out of the integral. Thus we see that the collapse rate for a particle moving with velocity $\mathbf{v}_{0}$, as compared to the collapse rate for the particle at rest, is smaller by the factor $M_{i} / E_{0}=\sqrt{1-\left(\mathbf{v}_{0} / c\right)^{2}}$, i.e., it is time dilated [31]. 


\section{CONCLUDING REMARKS}

In his famous 1964 paper [32] John Bell concluded:

"In a theory in which parameters are added to quantum mechanics to determine the results of individual measurements, without changing the statistical predictions, there must be a mechanism whereby the setting of one measurement device can influence the reading of another instrument, however remote. Moreover, the signal involved must propagate instantaneously, so that such a theory could not be Lorentz invariant."

In the collapse models discussed here, the additional parameters are the values of the fluctuating field $\mathrm{w}(\mathrm{x})$ at all spacetime points. The mechanism has two nonlocal aspects. One is the effect of the Probability Rule: it correlates field values at spacelike separated points. For example, this makes highly likely the survival of only one state describing a localized clump of particles when the statevector starts out in a superposition of widely separated clump states. The other is the effect of the direct product: it can correlate the states of two different widely separated systems. For example, if the statevector starts out as a sum of direct products, (an entangled state), the collapse suffered by one of the systems brings about the collapse of both to a single product.

In this paper we have seen that one can construct a Lorentz invariant collapse model. We have been led to considerations of tachyons so as to avoid infinite spontaneous particle production from the vacuum in lowest order while retaining CSL collapse behaviorin the nonrelativistic limit . In retrospect, one might have looked at Bell's second sentence cited above and realized that there is a natural Lorentz invariant nonlocal structure which can propagate instantaneously, and so be led to consider tachyons by another route.

The tachyonic structure seems remarkably well matched to the task: it is relativistic, it supplies the spacelike fourmomenta needed for collapse, it eliminates vacuum excitation to lowest order, it allows removal of time-ordering so as to eliminate vacuum excitation to all orders without giving a vacuous theory (as would its replacement by an ordinary particle, one with timelike four-momentum), it forces pair production and annihilation to disappear as kinematically untenable thus making the theory finite, it enables a model that is local to a high degree of accuracy. .

The present paper shows one way of obtaining a finite relativistic collapse model, so it may be considered as an existence proof. Elsewhere I shall consider other models that achieve this which use time-ordering, e.g., a model which employ a relativistically invariant cutoff to restrict energy available for vacuum excitation, and which may be applied to particles which interact.

Experiments have suggested mass proportional coupling of particles to the fluctuating field [8, 13,14$]$ which implies a connection to gravity. Interestingly, some string theory models conjoin the notions of tachyons and gravity. It appears that string theorists get rid of the tachyons for various good reasons. But perhaps tachyons do have a role to play in fundamental physics and, as Bell suggested [17] a connection exists between the resolution of the problem of infinities in quantum field theory as embodied in the string theory program and the resolution of the reality problem.

\section{ACKNOWLEDGMENTS}

I would like to dedicate this paper to the memory of my friend Euan Squires and to the memory of my friend and student Qijia Fu. I am in debt to Renata Grassi for stimulating conversations some time ago, and to Qijia Fu, Giancarlo Ghirardi, Adrian Kent, Euan Squires and Henry Stapp for helpful comments on previous versions of this paper.

\section{APPENDIX A: INDIVIDUAL STATEVECTOR COLLAPSE}

We illustrate here how collapse occurs for individual statevectors according to the generalized evolution equation (3.1). We use the example in section II A where, instead of the CSL Eq. (2.5a), Eq. (3.1) yields

$$
\left|\psi, T>=\sum_{i} c_{i}\right| n_{i}>e^{-\frac{1}{4 \lambda} \int_{T_{0}}^{T} d t d t^{\prime} d \mathbf{x} d \mathbf{x}^{\prime}\left[w(\mathbf{x}, t)-2 \lambda n_{i}(\mathbf{x})\right] G\left(x-x^{\prime}\right)\left[w\left(\mathbf{x}^{\prime}, t\right)-2 \lambda n_{i}\left(\mathbf{x}^{\prime}\right)\right]}
$$

As in section $\llbracket \mathrm{A}$, set $w(\mathbf{x}, t)=2 \lambda n_{i}$ to show how a $w(\mathbf{x}, t)$ which makes the exponential in the ith term large $(=1)$ can make small the jth exponential:

$$
e^{-\lambda \int d \mathbf{x} d \mathbf{x}^{\prime}\left[n_{i}(\mathbf{x})-n_{j}(\mathbf{x})\right] \int_{T_{0}}^{T} d t d t^{\prime} G\left(\mathbf{x}-\mathbf{x}^{\prime}, t-t^{\prime}\right)\left[n_{i}\left(\mathbf{x}^{\prime}\right)-n_{j}\left(\mathbf{x}^{\prime}\right)\right]}
$$

The time integrals in $(\mathrm{A2} 2)$ yield 


$$
2\left(T-T_{0}\right) \int_{0}^{T-T_{0}} d \tau G\left(\mathbf{x}-\mathbf{x}^{\prime}, \tau\right)-2 \int_{0}^{T-T_{0}} d \tau \tau G\left(\mathbf{x}-\mathbf{x}^{\prime}, \tau\right)
$$

The first term in $(\mathrm{A} 3)$ grows $\sim T$ while the second term eventually approaches a constant (assuming the integrals converge as $T \rightarrow \infty$ ). Thus, as in the example in section 1, the exponential (A2) approaches zero for $j \neq i$. If $w(\mathbf{x}, t)=2 \lambda n_{i}+w_{0}(\mathbf{x}, t)$, where $w_{0}$ almost always fluctuates uniformly about zero, the results are the same. In this way the terms in Eq. (A1) approach regions of disjoint support in $w$-space. When $w$ lies in the $i$ th region, $|\psi, T>\rightarrow \sim| n_{i}>$ according to (A1), while the integrated probability over the $i$ th region $\int D w P_{T}(w) \rightarrow\left|c_{i}\right|^{2}$.

\section{APPENDIX B: FOURIER TRANSFORM REPRESENTATION}

It is well known that the Fourier transform of a Gaussian is a Gaussian:

$$
e^{-\frac{1}{2 \alpha} \sum_{i, j=1}^{N}\left[w_{i}-a_{i}\right] G_{i j}\left[w_{j}-a_{j}\right]}=\int \frac{1}{\sqrt{\operatorname{det} G}} \prod_{i=1}^{N} \frac{d \eta_{i}}{\sqrt{2 \pi / \alpha}} e^{-\frac{\alpha}{2} \sum_{i, j=1}^{N} \eta_{i} G_{i j}^{-1} \eta_{j}} e^{i \sum_{i=1}^{N} \eta_{i}\left[w_{i}-a_{i}\right]}
$$

where $G^{-1} G=1$. Applying this to Eq. (3.1), with

$$
\begin{aligned}
\alpha & \rightarrow 2 \gamma \\
G_{i j} & \rightarrow \sqrt{d x d x^{\prime}} G\left(x-x^{\prime}\right) \\
w_{i}-a_{i} & \rightarrow \sqrt{d x}[w(x)-2 \gamma F(x)] \\
\eta_{i} & \rightarrow \sqrt{d x} \eta(x) \\
G_{i j}^{-1} & \rightarrow G_{T_{0}, T}^{-1}\left(x, x^{\prime}\right) \\
D \eta & \equiv\left[\operatorname{det} \sqrt{d x d x^{\prime}} G\left(x-x^{\prime}\right)\right]^{-\frac{1}{2}} \prod_{\mathbf{x}, t=T_{0}}^{T} d \eta(x) \sqrt{\gamma / \pi}
\end{aligned}
$$

yields:

$$
\left|\psi, T>=\mathcal{T} \int D \eta e^{-\gamma \int_{T_{0}}^{T} d x d x^{\prime} \eta(x) G_{T_{0}, T}^{-1}\left(x, x^{\prime}\right) \eta\left(x^{\prime}\right)} e^{i \int_{T_{0}}^{T} d x \eta(x)[w(x)-2 \gamma F(x)]}\right| \psi, T_{0}>
$$

where

$$
\int_{T_{0}}^{T} d z G(x-z) G_{T_{0}, T}^{-1}\left(z, x^{\prime}\right)=\delta\left(x-x^{\prime}\right)
$$

As appears in Eq. (B3), $G_{T_{0}, T}^{-1}\left(x, x^{\prime}\right)$ is the inverse of $G\left(x-x^{\prime}\right)$ over the interval $\left(T_{0}, T\right)$.

If $\left(T_{0}, T\right)$ is $(-\infty, \infty)$, then $G^{-1}$ can be written simply in terms of $\tilde{G}(k)$, the Fourier transform of G:

$$
G^{-1}\left(x-x^{\prime}\right)=\frac{1}{(2 \pi)^{4}} \int d^{4} k e^{i k \cdot\left(x-x^{\prime}\right)} \frac{1}{\tilde{G}(k)}
$$

This simpler form of $G^{-1}$ can be utilized even if $\left(T_{0}, T\right)$ is finite, since (B1) may also be used to write

$$
\left|\psi, T>=\mathcal{T} \int D \eta e^{-\gamma \int_{-\infty}^{\infty} d x d x^{\prime} \eta(x) G^{-1}\left(x-x^{\prime}\right) \eta\left(x^{\prime}\right)} e^{i \int_{T_{0}}^{T} d x \eta(x)[w(x)-2 \gamma F(x)]}\right| \psi, T_{0}>
$$

which differs from (B2) only in the limits of the Gaussian and in $D \eta \sim \prod_{\mathbf{x}, t=-\infty}^{\infty} d \eta(x)$. When $\eta(x)$ in (B5) is integrated over for $-\infty<t<T_{0}$ and $T<t<\infty$, the result is (B2). The discrete analog of this can be seen from Eq. (B1) if we let the discrete indices go from $-\infty$ to $\infty$ but let $w_{i}-a_{i}=0$ for $i<1$ and $i>N$. In this case $G^{-1}$ is the inverse of $G$ in the infinite dimensional index space. When the integrals over $\eta_{i}$ for $i<1$ and $i>N$ are performed, Eq. (B1) is recovered.

Eqs. (B4), (B5) are written down on the supposition that $G^{-1}\left(x-x^{\prime}\right)$ exists. However, we will be applying them when $\tilde{G}(k)$ vanishes in large enough regions of $k$-space so that the integral (B4) does not exist. In this case one may 
replace $\tilde{G}(k)$ by a function $\tilde{G}_{\epsilon}(k)$ which approaches $\tilde{G}(k)$ as $\epsilon \rightarrow 0$ and which does exist, and take the limit $\epsilon \rightarrow 0$ after all calculations are completed. Alternatively, one may use the form (B2) with $G_{T_{0}, T}^{-1}\left(x, x^{\prime}\right)$ which generally does exist. In practice one doesn't have to worry about these niceties since perturbation expressions involve $G$ and not $G^{-1}$. Thus formal manipulations suffice to give sensible answers.

The Fourier transform representation (3.5) of the density matrix may be derived by applying (B1) to the density matrix expression (3.3). More easily, we may use the statevector representation (B1) in $\rho(T)=\int D w|\psi, T><\psi, T|$ : integration over $w(x)$ for each $x$ gives rise to a term $\sim \prod_{x} \delta\left(\eta(x)-\eta^{\prime}(x)\right)$, and integration over $\eta^{\prime}(x)$ for each $x$ results in $(3.5)$.

\section{APPENDIX C: LORENTZ INVARIANCE}

We wish to show that the statevector evolution (3.1)

$$
\left|\psi, \sigma>=\mathcal{T} e^{-\frac{1}{4 \gamma} \int_{\sigma_{0}}^{\sigma} d x d x^{\prime}[w(x)-2 \gamma F(x)] G\left(x-x^{\prime}\right)\left[w\left(x^{\prime}\right)-2 \gamma F\left(x^{\prime}\right)\right]}\right| \psi, \sigma_{0}>
$$

and the probability rule (2.2) describe a Lorentz invariant theory. For $\operatorname{RCSL}_{1}$, where $G\left(x-x^{\prime}\right)=\delta\left(x-x^{\prime}\right)$, it has been shown that the theory is Lorentz invariant [19]. However, $\mathrm{RCSL}_{1}$ possesses the Markovian property that two successive transformations from $\sigma_{0}$ to $\sigma_{1}$ and from $\sigma_{1}$ to $\sigma_{2}$ are equivalent to one transformation from $\sigma_{0}$ to $\sigma_{2}$, and this is a property not possessed in the more general case.

We first discuss Lorentz invariance from the passive point of view, and show that the evolution of $\mid \psi, \sigma_{0}>$ to $|\psi, \sigma\rangle$ is described identically in all Lorentz frames. Consider a Lorentz frame with coordinates $\bar{x}=L x$. Since $\bar{F}(\bar{x})=F(x), \bar{w}(\bar{x})=w(x), d \bar{x}=d x$, and $G\left(\bar{x}-\bar{x}^{\prime}\right)=G\left(x-x^{\prime}\right)$, we see that (C1) is equal to the same expression when unbarred quantities are replaced by barred quantities (the hypersurfaces $\sigma_{0}, \sigma$ are not changed although, of course, their expressions in terms of $x$ and $\bar{x}$ differ). Finally, the probability density $\langle\psi, \sigma \mid \psi, \sigma\rangle$ is invariant, as is the functional integration element $D \bar{w}(\bar{x})=D w(x)$.

Next we discuss Lorentz invariance from te active point of view and show that, if $U$ is the unitary transformation corresponding to $L^{-1}$, then

$$
U\left|\psi, \sigma>=\mathcal{T} e^{-\frac{1}{4 \gamma} \int_{\bar{\sigma}_{0}}^{\bar{\sigma}} d x d x^{\prime}\left[w^{\prime}(x)-2 \gamma F(x)\right] G\left(x-x^{\prime}\right)\left[w^{\prime}\left(x^{\prime}\right)-2 \gamma F\left(x^{\prime}\right)\right]} U\right| \psi, \sigma_{0}>
$$

Operating upon (C1) with $U$ replaces $\mid \psi, \sigma>$ by $U|\psi, \sigma>,| \psi, \sigma_{0}>$ by $U \mid \psi, \sigma_{0}>$, and $F(x)$ by $U F(x) U^{-1}=\bar{F}(x)$. Next, change the labels from $x$ to $\bar{x}$ and $x^{\prime}$ to $\bar{x}^{\prime}$. This is not a Lorentz transformation, but a consistent notation requires changing the integration limits from $\left(\sigma_{0}, \sigma\right)$ to $\left(\bar{\sigma}_{0}, \bar{\sigma}\right)$. (For example, if $\left(\sigma_{0}, \sigma\right)=\left(T_{0}, T\right)$ are the integration limits of $t$, and $L$ is the time translation $\bar{t}=t-\tau$, then a relabelling $t \rightarrow \bar{t}$ gives $\left(T_{0}, T\right)$ as the integration limits for $\bar{t}$, which are different hyperplanes than $t=\left(T_{0}, T\right)$.) Now we Lorentz transform back to the unbarred frame, using $\bar{F}(\bar{x})=F(x), d \bar{x}=d x$ and $G\left(\bar{x}-\bar{x}^{\prime}\right)=G\left(x-x^{\prime}\right)$, obtaining (C2), with $w^{\prime}(x) \equiv w(L x)$. Although $w^{\prime}(x)$ is not equal to $w(x)$, that does not matter since $w(x)$ is an arbitrary classical field, so the sets $\{w(x)\},\left\{w^{\prime}(x)\right\}$ are equivalent. Indeed, this is all that is necessary for the probability rule to be invariant.

\section{APPENDIX D: VACUUM EXCITATION TO ORDER $\gamma$ IN RCSL . $_{1}$}

We calculate the contribution of the diagrams $2 \mathrm{a}, \mathrm{b}, \mathrm{c}$ to the density matrix at "large" time $T / 2$ (i.e., $T>>\hbar / \mu c^{2} \approx$ $10^{-15} \mathrm{sec}$ ). With the initial state as the vacuum state, Eq. (4.1) (with the help of (4.4)) gives to lowest order

$$
\rho(T / 2)=|0><0|-\gamma \int_{-T / 2}^{T / 2} d x d x^{\prime} G\left(x-x^{\prime}\right) \mathcal{T}\left[\phi(x)\left[\phi\left(x^{\prime}\right),|0><0|\right]\right]
$$

Using Wick's theorem, and remembering that $\mathcal{T}$ means operators to the right of $|0><0|$ are time-reversed, yields

$$
\begin{aligned}
\rho(T / 2)=|0><0|-\gamma \int_{-T / 2}^{T / 2} d x d x^{\prime} G\left(x-x^{\prime}\right) \\
\cdot\left[\frac{1}{(2 \pi)^{3}} \int d k e^{i k \cdot\left(x-x^{\prime}\right)} \delta\left(k^{2}-\mu^{2}\right)|0><0|-2 \phi_{-}(x)|0><0| \phi_{+}\left(x^{\prime}\right)\right]
\end{aligned}
$$


where $\phi_{+}(x), \phi_{-}(x)$ are respectively the positive and negative frequency parts of $\phi$. The first term in the bracket of (D2) corresponds to the sum of the diagrams 2a,b, with $\delta\left(k^{2}-\mu^{2}\right)$ coming from the sum of the $\phi$ propagator and its complex conjugate. The last term in the bracket corresponds to $2 \mathrm{c}$. When we perform the spacetime integrals, we obtain

$$
\rho(T / 2)=|0><0|-\gamma \tilde{G}\left(\mu^{2}\right) T \int \frac{d \mathbf{k}}{\omega(\mathbf{k})}\left[\frac{V}{(2 \pi)^{3}}|0><0|-a^{\dagger}(\mathbf{k})|0><0| a(\mathbf{k})\right]
$$

(we have used $2 \pi \delta(\omega=0)=\int_{-T / 2}^{T / 2} d t \exp i 0 t=T$, and similarly $(2 \pi)^{3} \delta(\mathbf{k}=0)=V$ ).

The mean energy $\bar{H}(T / 2) \equiv \operatorname{Tr} \int d \mathbf{k} \omega(\mathbf{k}) a^{\dagger}(\mathbf{k}) a(\mathbf{k}) \rho(T / 2)$ is found using (D3) to be

$$
\bar{H}(T / 2)=\gamma \tilde{G}\left(\mu^{2}\right) T V \frac{1}{(2 \pi)^{3}} \int d \mathbf{k}
$$

It is apparent from Eq. (D4) that the energy/sec-vol produced out of the vacuum is infinite (as in $\mathrm{RCSL}_{1}$, where $\tilde{G}=1$ ) unless $\tilde{G}\left(\mu^{2}\right)=0$. Then, according to Eq. (D3), the vacuum remains the vacuum in order $\gamma$.

\section{APPENDIX E: NONLOCAL TRANSPORT IN RCSL}

\section{No-Time-Ordering Expressed As Time-Ordering} [33]:

We may express the not-time-ordered evolution as a time-ordered evolution. The following is a well-known identity

$$
\frac{d}{d t} e^{A(t)}=\left\{\int_{0}^{1} d \alpha e^{\alpha[A(t) \otimes 1-1 \otimes A(t)]} \frac{d A(t)}{d t}\right\} e^{A(t)}
$$

Its integral

$$
e^{A(T)}=\mathcal{T} e^{\int_{0}^{T} d t \int_{0}^{1} d \alpha e^{\alpha[A(t) \otimes 1-1 \otimes A(t)]} \frac{d A(t)}{d t}}
$$

(assuming $A\left(T_{0}\right)=0$ ) is the relation we need. Put

$$
A(t)=-i 2 \gamma(2 M) \int_{T_{0}}^{t} d x^{\prime} \eta\left(x^{\prime}\right): \psi_{t}^{2}\left(x^{\prime}\right):
$$

into (E2). (By $\psi_{t}\left(x^{\prime}\right)$ we mean $\psi\left(x^{\prime}\right)$ for all purposes except time-ordering, in which case it should be regarded as a function of $t$ : this is required by Eqs. (E2, E3), since $A(t)$ must be regarded as a function of $t$ for time-ordering.) Thus we obtain the unitary operator part of the integrands in Eqs. (7.3, 7.4 which has no time ordering, written in a time-ordered way:

$$
\begin{gathered}
e^{-i 4 \gamma M \int_{T_{0}}^{T} d x \eta(x) \psi_{t}^{2}\left(x^{\prime}\right)}=\mathcal{T} e^{-i \int_{T_{0}}^{T} d t H_{\text {eff }}(t)} \\
\int_{T_{0}}^{T} d t H_{e f f}(t) \equiv 4 \gamma M \int_{0}^{1} d \alpha \int_{T_{0}}^{T} d x \eta(x) e^{-i 4 \gamma M \alpha \int_{T_{0}}^{t} d x^{\prime} \eta\left(x^{\prime}\right)\left[: \psi_{t}^{2}\left(x^{\prime}\right): \otimes 1-1 \otimes: \psi_{t}^{2}\left(x^{\prime}\right):\right]}: \psi^{2}(x): \\
=4 \gamma M \int_{T_{0}}^{T} \eta(x): \psi^{2}(x): \\
+(4 \gamma M)^{2} \int_{T_{0}}^{T} d x \int_{T_{0}}^{t} d x^{\prime} \eta(x) \eta\left(x^{\prime}\right) i\left[\psi(x), \psi\left(x^{\prime}\right)\right]\left\{\psi(x), \psi_{t}\left(x^{\prime}\right)\right\}_{+} \\
+\frac{(4 \gamma M)^{3}}{3} \int_{T_{0}}^{T} d x \int_{T_{0}}^{t} d x^{\prime} d x^{\prime \prime} \eta(x) \eta\left(x^{\prime}\right) \eta\left(x^{\prime \prime}\right) i\left[\psi(x), \psi\left(x^{\prime}\right)\right] \cdot \\
\quad\left[i\left[\psi(x), \psi\left(x^{\prime \prime}\right)\right]\left\{\psi_{t}\left(x^{\prime}\right), \psi_{t}\left(x^{\prime \prime}\right)\right\}_{+}+i\left[\psi\left(x^{\prime}\right), \psi\left(x^{\prime \prime}\right)\right]\left\{\psi(x), \psi_{t}\left(x^{\prime \prime}\right)\right\}_{+}\right]+\ldots
\end{gathered}
$$


All terms in Eq. (E5b) are quadratic in the operator $\psi$ (the commutators are c-numbers). The first term is local. The remaining terms act like a nonlocal potential: a particle may be transported from $x$ to $x^{\prime}$. The second term, of order $\gamma^{2}$, vanishes for spacelike $x-x^{\prime}$ so, although it is nonlocal, it describes timelike transport. However, the transport may be in the backwards time direction and, when iterated, a sequence of timelike forward and backward motion may allow spacelike transport. The third and higher order terms, may also allow spacelike transport, due to a succession of commutators which have support in the forward and backward light cones. However, this discussion does not take into account the nature of the field $\eta(x)$ which appears in these expressions. Whether spacelike transport actually occurs in any term requires further investigation.

\section{Particle Propagation}

Here we calculate to lowest order the propagation of a particle initially located near the origin (say $|\mathbf{x}|<\sigma$ ) and with a fairly well-defined momentum, corresponding to diagram $4 \mathrm{c}$, for a finite time $T$. We take a pragmatic approach to the problems of defining a position operator and a localized wavefunction in a relativistic theory. We use the usual (nonrelativistic) position operator $\mathbf{X}$ with eigenstates $|\mathbf{x}\rangle$, choose the wavepacket width $<<M^{-1}$, and regard a wavefunction's exponential decay outside the light cone (with characteristic length $M^{-1}$ ) as normal, i.e., as not indicative of superluminal transport.

The desired probability is given by Eq. (7.5) as:

$$
\sim \gamma M^{2} \int_{0}^{T} d x d x^{\prime} G\left(x-x^{\prime}\right)<\mathbf{z}, T\left|\psi_{-}\left(x^{\prime}\right) \psi_{+}\left(x^{\prime}\right)\right| \Phi><\Phi\left|\psi_{-}(x) \psi_{+}(x)\right| \mathbf{z}, T>
$$

In this and what follows we shall drop purely numerical factors. Here, $<\mathbf{k} \mid \Phi>$ is the initial wavefunction in momentum space and $|\mathbf{z}, T\rangle$ is an eigenstate of the interaction picture position operator $\mathbf{X}(T)(<\mathbf{z}, T|\mathbf{k}\rangle=\exp i(\mathbf{k} \cdot \mathbf{z}-E T))$. After some integrations we obtain

$$
\begin{aligned}
\sim \gamma M^{2} \int \frac{d \mathbf{p} d \mathbf{k} d \mathbf{k}^{\prime} e^{i\left(\mathbf{k}-\mathbf{k}^{\prime}\right) \cdot \mathbf{z}-i\left(E_{\mathbf{k}+\mathbf{p}}-E_{\mathbf{k}^{\prime}+\mathbf{p}}\right) T}}{\epsilon_{\mathbf{p}} \sqrt{E_{\mathbf{k}} E_{\mathbf{k}+\mathbf{p}} E_{\mathbf{k}^{\prime}} E_{\mathbf{k}^{\prime}+\mathbf{p}}}}<\mathbf{k}|\Phi><\Phi| \mathbf{k}^{\prime}> \\
\cdot \sum_{s=-1}^{1} \int_{0}^{T} \int_{0}^{T} d t d t^{\prime} e^{i\left[E_{\mathbf{k}+\mathbf{p}}-E_{\mathbf{k}}-s \epsilon_{\mathbf{p}}\right] t} e^{-i\left[E_{\mathbf{k}^{\prime}+\mathbf{p}}-E_{\mathbf{k}^{\prime}}-s \epsilon_{\mathbf{p}}\right] t^{\prime}}
\end{aligned}
$$

where $\epsilon_{\mathbf{p}} \equiv \sqrt{\mathbf{p}^{2}-\mu^{2}}, E_{\mathbf{k}} \equiv \sqrt{\mathbf{k}^{2}+M^{2}}$.

We may use one of the time integrals to give energy conservation:

$$
\int_{0}^{T} d t e^{i \Delta E t}=\frac{\sin (\Delta E T)}{\Delta E}+2 i \frac{\sin ^{2}(\Delta E T / 2)}{\Delta E} \approx \frac{\pi}{2} \delta(\Delta E)+2 i \mathcal{P} \frac{1}{\Delta E} \approx \frac{\pi}{2} \delta(\Delta E)
$$

The term $\mathcal{P} 1 / \Delta E$ is dropped in $(\mathrm{E} 8)$ as it is a well known artifact of the abrupt turning on and off of the interaction and can be eliminated by more careful attention to that detail. (E8) is a good approximation provided the rest of the integrand does not oscillate in energy with period greater than $\approx \hbar / T \approx 6 \cdot 10^{-16} \mathrm{eVsec} / T$. Even for a relatively short time such as $T \approx 10^{-14} \mathrm{sec}$ this gives energy conservation to better than $.1 \mathrm{eV}$ (which improves with increasing $\mathrm{T}$ ). (E8) can be used to integrate over $\epsilon_{\mathbf{p}}$ because the dependence of the rest of the integrand is upon $\mathbf{p}=\sqrt{\epsilon^{2}+\mu^{2}}$ which varies slowly over e.g., $\Delta \epsilon=.1 \mathrm{eV}$.

We shall also assume that $<\mathbf{k} \mid \Phi>\equiv \tilde{\Phi}\left(\mathbf{k}-\mathbf{k}_{0}\right)$ is peaked at $\mathbf{k}_{0}$. The kinematics of absorption/emission of a tachyon by a particle initially moving with momentum $\mathbf{k}$ can be shown to yield the tachyon momentum magnitude (which is the magnitude of the increase in particle momentum)

$$
|\mathbf{p}(\mathbf{k}, \hat{\mathbf{p}})|=\frac{E_{\mathbf{k}} \mu}{\sqrt{(\mathbf{k} \times \hat{\mathbf{p}})^{2}+M^{2}}}
$$

to o $(\mu / M)$ or better. Since $|\mathbf{p}|<<E_{\mathbf{k}}$, and if $\tilde{\Phi}$ 's peak is narrow, we can expand $E_{\mathbf{k}+\mathbf{p}}$ to second order in $\mathbf{p}$ and $\kappa \equiv \mathbf{k}-\mathbf{k}_{\mathbf{0}}$. (E7) then becomes (with replacement of $\mathbf{k}_{\mathbf{0}}$ by $\mathbf{k}$, and $E_{\mathbf{k}}$ by $E$ ):

$$
\sim \frac{\gamma M^{2}}{E^{2}} \int|\mathbf{p}(\mathbf{k}, \hat{\mathbf{p}})| d \Omega_{\mathbf{p}} d \kappa d \kappa^{\prime} e^{i\left(\kappa-\kappa^{\prime}\right) \cdot[\mathbf{z}-(\mathbf{v}+\mathbf{w})] T} \tilde{\Phi}(\kappa) \tilde{\Phi}^{*}\left(\kappa^{\prime}\right) \int_{0}^{T} d t e^{i\left(\kappa-\kappa^{\prime}\right) \cdot \mathbf{w} t}
$$




$$
\begin{aligned}
& \sim \frac{\gamma M^{2}}{E^{2}} \int|\mathbf{p}(\mathbf{k}, \hat{\mathbf{p}})| d \Omega_{\mathbf{p}} \int_{0}^{T} d t|\Phi(\mathbf{z}-\mathbf{v} T-\mathbf{w}(T-t))|^{2} \\
& \approx \frac{\gamma M^{2}}{E^{2}} \int|\mathbf{p}(\mathbf{k}, \hat{\mathbf{p}})| d \Omega_{\mathbf{p}} \int_{0}^{T} d t \delta[\mathbf{z}-\mathbf{v} T-\mathbf{w}(T-t)]
\end{aligned}
$$

where

$$
\mathbf{v} \equiv \frac{\mathbf{k}}{E} \quad \text { and } \quad \mathbf{w} \equiv \frac{\mathbf{p}}{E}-\frac{\mathbf{p} \cdot \mathbf{k k}}{E^{3}}
$$

are the initial velocity of the particle and its velocity gain respectively. In the integrand of (E10a), for simplicity we have omitted the factor exp $-i\left(\kappa^{2}-\kappa^{\prime 2}\right) T$ responsible for the usual spread of the wavepacket: we assume that $\sigma$ is large enough $(\sigma>a)$ so that this spread is less than the spread described by (E10). On the other hand, we assume that $\sigma<<$ the spread described by (E10), so that $\Phi^{2}$ can be well approximated by the delta function in (E10d).

In the nonrelativistic limit, $\mathbf{p}=\mu c \hat{\mathbf{p}}$ and $\mathbf{w}=(\mu c / M) \hat{\mathbf{p}}$ by Eqs. (E9), (E11). With the substitution $\hat{\mathbf{p}}(T-t) \equiv \mathbf{r}$, the integral over $\mathbf{r}$ in E10d is easily performed with the result:

$$
\sim \frac{\gamma M}{|\mathbf{z}-\mathbf{v} T|^{2}} \Theta\left[\frac{\mu}{M} c T-|\mathbf{z}-\mathbf{v} T|\right]
$$

( $\Theta$ is the step function.) This is precisely the same distribution as would be obtained from a collection of classical particles, all of which start at $t=0$ from $\mathbf{z}=0$ with velocity $\mathbf{v}$, each of which impulsively acquires momentum of magnitude $\mu c T / M$ in a random direction at an arbitrary time between 0 and $T$.

This result is used in section VIIIB to explain how a succession of such evolutions in sufficiently high order (and so with very small probability) will eventually go outside of the lightcone. We note here that the above calculation does not take into account the free backwards-in-time evolution because it calculates the wavefunction in the basis $\mid \mathbf{z}, T>$ and not in the basis $\mid \mathbf{z}, 0>$. The latter is the appropriate basis to use when iterating diagrams as it introduces no extra time evolution beyond what the expansion provides (i.e., it includes the free backwards-in-time evolution). The former is to be used to find the probability of the particle's position $(\mid \mathbf{z}, T>$ is an eigenstate of the interaction picture position operator) only at the end of the calculation of the contribution of each order, where its use correctly eliminates the final free backwards-in-time evolution.

With relativistic $\mathbf{k}$, first consider $\mathbf{k}$ parallel to $\hat{\mathbf{u}} \equiv[\mathbf{z}-\mathbf{v} T] /|\mathbf{z}-\mathbf{v} T|$. The delta function in (E10d) can only vanish for $\mathbf{w} \| \hat{\mathbf{u}}$ which, by (E11), implies that $\mathbf{p} \| \hat{\mathbf{u}}$. Then $|\mathbf{p}|=E \mu / M$ by (E9), and $\mathbf{w}=\hat{\mathbf{p}} \mu M / E^{2}$ by (E11). The integral in $(\mathrm{E} 10 \mathrm{~d})$ can then be performed with the result:

$$
\sim \frac{\gamma E}{|\mathbf{z}-\mathbf{v} T|^{2}} \Theta\left[\frac{\mu M}{E^{2}} c T-|\mathbf{z}-\mathbf{v} T|\right]
$$

With $\mathbf{k} \perp \hat{\mathbf{u}}$, then $\mathbf{k} \cdot \mathbf{w}=0$ ( since $\mathbf{w} \| \hat{\mathbf{u}})$ which implies $\mathbf{k} \cdot \mathbf{p}=0$ by (E11). Then $|\mathbf{p}|=\mu$ by $($ E9) and $\mathbf{w}=\hat{\mathbf{p}}(\mu / E)$ by (E11). (E100) then yields:

$$
\sim \frac{\gamma M^{2}}{|\mathbf{z}-\mathbf{v} T|^{2}} \Theta\left[\frac{\mu}{E} c T-|\mathbf{z}-\mathbf{v} T|\right]
$$

In the nonrelativistic limit, Eqs. (E13), (E14) agree with the result (E12). They describe (to accuracy o $(\mu / M))$ the diminution in the wavepacket spread which is the effect of a Lorentz transformation on the zero velocity spread..

As an interesting application of the relativistic results, consider the spreading of a wavepacket when one one starts with a particle at rest and in each order has all p's parallel (i.e., one does does not perform the integration over the p's). This accelerates the particle to an arbitrarily large energy but, as mentioned in section VIIIB, the result (see (E15)) is that the wavefunction does not go outside the lightcone. I shall not go into the details of how the successive iterations (the higher order terms) in the perturbation expansion work: in (E6) one must calculate the matrix element between $\left\langle\mathbf{z}, 0|\ldots| \mathbf{z}^{\prime}, 0\right\rangle$ in order to obtain the wavefunction appropriate for the next iteration, etc. The momentum gained by the particle with each successive iteration, given by ( $\mathrm{E9})$, is $\Delta k=E \mu / M$. The increase in spreading in the direction of the accumulating momentum, given by (E13), is $\Delta L=\Delta k M^{2} T / E^{3}$. Integrating over $k$ from 0 to $\infty$ gives the upper limit on the accumulated wavefunction spread for any number of iterations:

$$
L<c T \int_{0}^{\infty} d k \frac{M^{2}}{E^{3}}=c T
$$




\section{APPENDIX F: RELATIVISTIC ENERGY PRODUCTION TO ORDER $\gamma$.}

We calculate here $\bar{H}(T / 2) \equiv \operatorname{Tr} H \rho(T / 2)$, for an initial $n$ particle state $\rho(-T / 2)=\left|\Psi_{0}><\Psi_{0}\right|$. By Eq. (7.5),

$$
\begin{aligned}
\bar{H}(T / 2)-\bar{H}(-T / 2)=-2 \gamma(2 M)^{2} \int_{-\frac{T}{2}}^{\frac{T}{2}} d x d x^{\prime} G\left(x-x^{\prime}\right) \\
\cdot<\Psi_{0}\left|\left[\psi_{-}(x) \psi_{+}(x)\left[\psi_{-}\left(x^{\prime}\right) \psi_{+}\left(x^{\prime}\right), H\right]\right]\right| \Psi_{0}>
\end{aligned}
$$

Replacement of $\left[\psi_{-}\left(x^{\prime}\right) \psi_{+}\left(x^{\prime}\right), H\right]$ by $i \partial \psi_{-}\left(x^{\prime}\right) \psi_{+}\left(x^{\prime}\right) / \partial t^{\prime}$ followed by integration by parts over $t^{\prime}$ (the surface terms are exponentially small for large $T$, and may be neglected), and evaluation of the remaining commutator results in

$$
\begin{aligned}
\bar{H}(T / 2)-\bar{H}(-T / 2)=-4 i \gamma(2 M)^{2} \int_{-\frac{T}{2}}^{\frac{T}{2}} d x d x^{\prime} \frac{\partial}{\partial t} G\left(x-x^{\prime}\right) \\
\left.\cdot<0\left|\psi_{+}(x) \psi_{-}\left(x^{\prime}\right)\right| 0><\Psi_{0} \mid \psi_{-}(x) \psi_{+}\left(x^{\prime}\right)\right) \mid \Psi_{0}>
\end{aligned}
$$

Next, the integral over $x, x^{\prime}$ gives

$$
\begin{aligned}
\bar{H}(T / 2)-\bar{H}(-T / 2)=\frac{1}{\pi^{2}} \gamma(2 M)^{2} \int d k \frac{d \mathbf{p}}{2 E} \frac{d \mathbf{p}_{1}}{\sqrt{2 E_{1}}} \frac{d \mathbf{p}_{2}}{\sqrt{2 E_{2}}} \\
\cdot k^{0} \delta\left(k^{2}+\mu^{2}\right) \delta\left(k-p+p_{1}\right) \delta\left(k-p+p_{2}\right)<\Psi_{0}\left|a^{\dagger}\left(\mathbf{p}_{1}\right) a\left(\mathbf{p}_{2}\right)\right| \Psi_{0}>
\end{aligned}
$$

We may set $\delta\left(p_{1}-p_{2}\right)=\delta\left(\mathbf{p}_{1}-\mathbf{p}_{2}\right) T / 2 \pi$ and perform the integral over $p_{2}$ :

$$
\begin{aligned}
\bar{H}(T / 2)-\bar{H}(-T / 2)= & \frac{1}{2 \pi^{3}} \gamma T(2 M)^{2} \int \frac{d \mathbf{p}_{1}}{2 E_{1}}<\Psi_{0}\left|a^{\dagger}\left(\mathbf{p}_{1}\right) a\left(\mathbf{p}_{1}\right)\right| \Psi_{0}> \\
& \left.\cdot \int d k k^{\nu} \delta\left(k^{2}+\mu^{2}\right) \int \frac{d \mathbf{p}}{2 E} \delta\left(k-p+p_{1}\right)\right|_{\nu=0}
\end{aligned}
$$

The integral expression in the last line of $(\sqrt{\mathrm{F} 4})$ is a four-vector function of $p_{1}$, so it is equal to $p_{1}^{\nu} C$, where $C$ is a constant. The zeroth component of this four-vector is $E_{1} C$, so the integral over $\mathbf{p}_{1}$ yields the particle number operator, whose matrix element is $n$. Thus (F4) becomes

$$
\bar{H}(T / 2)-\bar{H}(-T / 2)=\frac{1}{\pi^{3}} n \gamma T M^{2} C
$$

$\mathrm{C}$ is most easily evaluated in the Lorentz frame in which $p_{1}=(M, \mathbf{0})$ :

$$
M C=\int \frac{d \mathbf{k}}{2 \sqrt{\mathbf{k}^{2}+\mu^{2}}} d k^{0} k^{0} \delta\left(k^{2}+\mu^{2}\right) \delta\left(k^{0}-\sqrt{\mathbf{k}^{2}+M^{2}}+M\right)=M \frac{\pi}{2}\left(\frac{\mu}{M}\right)^{3} \sqrt{1+\left(\frac{\mu}{2 M}\right)^{2}}
$$

Eqs. (F5) and ( $\mathrm{F6}$ ) result in Eq. (9.2).

\section{APPENDIX G: RELATIVISTIC COLLAPSE RATE TO ORDER $\gamma$.}

Consider a particle in the initial state $\alpha|L>+\beta| R>$, where $\mid L>$ is a reasonably well localized state around $\mathbf{x}=\mathbf{L}$ and, for simplicity, $\mid R>$, is the same state translated by a large distance to $\mathbf{R}$, with $|\mathbf{L}-\mathbf{R}|>>c T$. Since $\psi_{-}(\mathbf{x}, 0) \psi_{+}(\mathbf{x}, 0) \mid L>$ is a state still localized in the region around $\mathbf{L}$ (the operator is a kind of particle number density operator smeared over the distance $M^{-1}$ ) then, even when t is not 0 ,

$$
<R\left|\psi_{-}(x) \psi_{+}(x)\right| L>\approx 0, \quad<R\left|\psi_{-}(x) \psi_{+}(x) \psi_{-}\left(x^{\prime}\right) \psi_{+}\left(x^{\prime}\right)\right| L>\approx 0
$$

by our hypothesis of large separation of the states. This helps simplify the density matrix expression $(7.5)$ : 


$$
\begin{gathered}
<L|\rho(T / 2)| R>=\alpha \beta^{*}\left\{1-2 \gamma(2 M)^{2} \int d x d x^{\prime} G\left(x-x^{\prime}\right)\right. \\
\cdot\left[<L\left|\psi_{-}(x) \psi_{+}(x) \psi_{-}\left(x^{\prime}\right) \psi_{+}\left(x^{\prime}\right)\right| L>+<R\left|\psi_{-}(x) \psi_{+}(x) \psi_{-}\left(x^{\prime}\right) \psi_{+}\left(x^{\prime}\right)\right| R>\right. \\
\left.\left.\quad-2<L\left|\psi_{-}(x) \psi_{+}(x)\right| L><R\left|\psi_{-}\left(x^{\prime}\right) \psi_{+}\left(x^{\prime}\right)\right| R>\right]\right\}
\end{gathered}
$$

The first two terms in the square bracket in (G1) correspond to the Feynman diagrams in Fig. 4a, the last term to Fig. 4c.

The last term in the bracket of (G1) has the form $f(\mathbf{x}-\mathbf{L}, t) f\left(\mathbf{x}^{\prime}-\mathbf{R}, t^{\prime}\right)$ where $f(\mathbf{z}, 0)$ is fairly well localized, around $\mathbf{z}=0 . G$ decreases sufficiently rapidly (see Eqs. (5.4)) so that the contribution of the last term vanishes as $|\mathbf{L}-\mathbf{R}| \rightarrow \infty$. Because of translation invariance, the first two matrix elements in the bracket of (G1) are identical. After commuting the two middle operators in the matrix element (the commutator gives no contribution because its support vanishes where $G$ 's does not), we obtain

$$
\begin{aligned}
<L|\rho(T / 2)| R>=\alpha \beta^{*}\left\{1-4 \gamma(2 M)^{2} \int d x d x^{\prime} G\left(x-x^{\prime}\right)\right. & \\
\cdot & \left.<0\left|\psi_{+}(x) \psi_{-}\left(x^{\prime}\right)\right| 0><L\left|\psi_{-}(x) \psi_{+}\left(x^{\prime}\right)\right| L>\right\}
\end{aligned}
$$

We next perform the integrals over $x$ and $x^{\prime}$ :

$$
\begin{aligned}
& <L|\rho(T / 2)| R>=\alpha \beta^{*}\left\{1-\frac{1}{\pi^{2}} \gamma(2 M)^{2} \int d k \frac{d \mathbf{p}}{2 E} \frac{d \mathbf{p}_{\mathbf{1}}}{\sqrt{2 E_{1}}} \frac{d \mathbf{p}_{\mathbf{2}}}{\sqrt{2 E_{2}}}\right. \\
& \left.\cdot \delta\left(k^{2}+\mu^{2}\right) \delta\left(k-p+p_{1}\right) \delta\left(p_{1}-p_{2}\right)<L\left|a^{\dagger}\left(\mathbf{p}_{\mathbf{2}}\right)\right| 0><0\left|a\left(\mathbf{p}_{\mathbf{1}}\right)\right| L>\right\}
\end{aligned}
$$

We shall write the normalized wavefuction in momentum space as $<0\left|a\left(\mathbf{p}_{\mathbf{1}}\right)\right| L>\equiv \Psi\left(\mathbf{p}_{\mathbf{1}}\right)$. Since $\delta\left(p_{1}-p_{2}\right)=$ $\delta\left(\mathbf{p}_{\mathbf{1}}-\mathbf{p}_{\mathbf{2}}\right) T / 2 \pi$, we get

$$
<L|\rho(T / 2)| R>=\alpha \beta^{*}\left\{1-\frac{1}{2 \pi^{3}} \gamma T(2 M)^{2} \int \frac{d \mathbf{p}_{\mathbf{1}}}{2 E_{1}}\left|\Psi\left(\mathbf{p}_{\mathbf{1}}\right)\right|^{2} \int d k \delta\left(k^{2}+\mu^{2}\right) \int \frac{d \mathbf{p}}{2 E} \delta\left(k-p+p_{1}\right)\right\}
$$

The last two integrals in (G4) are a Lorentz scalar function of $p_{1}^{2}=M^{2}$, i.e., they do not depend upon $p_{1}^{\mu}$ at all. The integrals are most easily performed in the frame in which $p_{1}=(M, \mathbf{0})$ :

$$
\int d k \delta\left(k^{2}+\mu^{2}\right) \int \frac{d \mathbf{p}}{2 E} \delta\left(k-p+p_{1}\right)=\pi \frac{\mu}{M} \sqrt{1+\left(\frac{\mu}{2 M}\right)^{2}}
$$

Thus we obtain the off-diagonal elements of the density matrix as

$$
<L|\rho(T / 2)| R>=\alpha \beta^{*}\left\{1-\frac{2}{\pi^{2}} \gamma T \mu M \sqrt{1+\left(\frac{\mu}{2 M}\right)^{2}} \int \frac{d \mathbf{p}}{2 E}|\Psi(\mathbf{p})|^{2}\right\}
$$

While some of the depletion of the initial states $\mid L>$ and $\mid R>$ is due to their excitation and not collapse, Eq. (G6) still serves to give a good measure of the collapse rate.

[1] An incomplete sample is: E.P. Wigner, American Journal of Physics 31, 6 (1963); P. Pearle, Amer. Journ of Phys. 35, 742 (1967); L. E. Ballentine, Revs. Mod. Phys. 42, 385 (1970); B. d'Espagnat, Conceptual Foundations of Quantum Mechanics, (W. A. Benjamin, Menlo Park, Cal. 1971); F. J. Belinfante, A Survey of Hidden-Variable Theories, (Pergamon, Oxford 1973); J. S. Bell in Sixty-Two Years of Uncertainty, edited by A. Miller (Plenum, New York 1990), p.17; D. Z. Albert, Quantum Mechanics and Experience, (Harvard, Cambridge 1994).

[2] D. Bohm and J. Bub, Revs. Mod. Phys. 38, 453 (1966). 
[3] P. Pearle, Phys. Rev. D 13, 857 (1976); Int'l. Journ. Theor. Phys. 48, 489 (1979); Found. Phys. 12, 249 (1982); Phys. Rev. D 29, 235 (1984); in The Wave-Particle Dualism, edited by S. Diner et. al (Reidel, Dordrecht 1984); Journ. Stat. Phys. 41, 719 (1985); in Quantum Concepts in Space and Time, edited by R. Penrose and C. J. Isham (Clarendon, Oxford, 1986); Phys. Rev. D 33, 2240 (1986); in New Techniques in Quantum Measurement Theory, edited by D. M. Greenberger (N.Y. Acad. of Sci., N.Y., 1986), p.539; N. Gisin, Phys. Rev. Lett. 52, 1657 (1984).

[4] G. C. Ghirardi, A. Rimini and T. Weber, Phys. Rev. D 34, 470 (1986); Phys. Rev. D 36, 3287 (1987); Found. Phys. 18, $1,(1988)$.

[5] J. S. Bell in Schrodinger-Centenary celebration of a polymath, edited by C. W. Kilmister (Cambridge University Press, Cambridge 1987) and in Speakable and unspeakable in quantum mechanics, (Cambridge University Press, Cambridge 1987).

[6] P. Pearle, Phys. Rev. A 39, 2277 (1989)

[7] G. C. Ghirardi, P. Pearle and A. Rimini, Phys. Rev. A 42, 78 (1990).

[8] P. Pearle and E. Squires, Phys. Rev. Lett. 73, 1 (1994).

[9] Suggested by John Clauser (private communication).

[10] P. Pearle, Phys. Rev. D 29, 235 (1984); A. Zeilinger in Quantum Concepts in Space and Time, edited by R. Penrose and C. J. Isham (Clarendon, Oxford, 1986), p.16; A. Zeilinger, R. Gaehler, C. G. Shull, W. Treimer and W. Mampe, Revs. Mod. Phys. 60, 1067 (1988).

[11] G. C. Ghirardi and A. Rimini in Sixty-Two Years of Uncertainty, edited by A. Miller (Plenum, New York 1990), p.167.

[12] E. J. Squires, Phys. Lett. A 158, 431 (1991)

[13] B. Collett, P. Pearle, F. Avignone and S. Nussinov, Found. Phys. 25, 1399 (1995);

[14] P. M. Pearle, J. Ring, J. I. Collar and F. T. Avignone III, Found. Phys. (to be published).

[15] F. Karolyhazy, Nuovo Cimento 42A, 1506 (1966); F. Karolyhazy, A Frenkel and B. Lukacs in Physics as Natural Philosophy, edited by A. Shimony and H. Feshbach (M.I.T. Press, Cambridge 1982), p. 204; in Quantum Concepts in Space and Time, edited by R. Penrose and C. J. Isham (Clarendon, Oxford 1986), p. 109; A. Frenkel, Found. Phys. 20, 159 (1990); R. Penrose in Quantum Concepts in Space and Time, edited by R. Penrose and C. J. Isham (Clarendon, Oxford 1986), p. 129; in The Emperor's New Mind, (Oxford University Press, Oxford, 1992); in Shadows of the Mind, (Oxford University Press, Oxford, 1994).

[16] L. Diosi, Phys. Rev. A 40, 1165 (1989); G. C. Ghirardi, R. Grassi and A. Rimini, Phys. Rev. A 42, 1057 (1990); P. Pearle and E. J. Squires, Found. Phys. 26, 291 (1996).

[17] J. S. Bell in Themes in Contemporary Physics II, Essays in honor of Julian Schwinger's 70th birthday, edited by S. Deser and R. J. Finkelstein (World Scientific, Singapore 1989), p.1.

[18] P. Pearle, in Sixty-Two Years of Uncertainty, edited by A. Miller, (Plenum, New York 1990), p. 193.

[19] G. C. Ghirardi, R. Grassi and P. Pearle, Found. Phys. 20, 1271 (1990); in Proceedings of the Symposium on the Foundations of Modern Physics 1990, edited by P. Lahti and P. Mittelstaedt, (World Scientific, Singapore 1991), p. 109.

[20] P. Pearle in Quantum Chaos-Quantum Measurement, edited by P. Cvitanovic et. al., (Kluwer, the Netherlands 1992), p.283.

[21] N. Dowrick, "Path integrals and the GRW model" (preprint, Oxford 1993).

[22] P. Pearle, Phys. Rev. A 48, 913 (1993); in Stochastic Evolution of Quantum States in Open Systems and in Measurement Processes, edited by L. Diosi and B. Lukacs (World Scientific, Singapore 1994), p. 79; in Perspectives on Quantum Reality, edited by R. Clifton (Kluwer, Dordrecht 1996), p. 93.

[23] P. T. Cox, The Algebra Of Probable Inference (Johns Hopkins, Baltimore 1961); see also R. Baierlein, Atoms And Information Theory (Freeman, San Francisco 1971) for a discussion of probability as used here, as a measure of rational belief based upon the available evidence, applied to statistical physics.

[24] G. C. Ghirardi and P. Pearle in PSA 1990, Vol.2, A. Fine, M. Forbes and L. Wessels eds. (Philosophy of Science Association, East Lansing, Michigan 1990).

[25] G. C. Ghirardi and R. Grassi, Stud. Hist. Philos. Sci. 25, 97 (1994).

[26] G. C. Ghirardi, Found. Phys. Lett. 9, 313 (1996).

[27] Y. Aharonov and D. Z. Albert, Phys. Rev. D 21, 3316 (1980); Phys. Rev. D 24, 359 (1981); Phys. Rev. D 29, 228 (1984).

[28] T. Weber, Nuovo Cimento 106B, 1111 (1991).

[29] G. Feinberg, Phys. Rev. 159, 1089 (1967).

[30] A few years ago, when I told Renata Grassi about my attempts at this new formalism, she suggested that it then might be worthwhile to try again to directly couple $w(\mathbf{x}, t)$ to the nucleon number density, without the intermediacy of the $\phi$-particle. At the time we did not appreciate that the two models could be equivalent in the sense shown here.

[31] Long before this calculation was possible, Renata Grassi (private communication) argued that, since one would expect the radiation rate of an atom due to excitation by collapse (calculated by nonrelativistic CSL) to be time dilated when the atom is moving, one could conclude that the collapse rate itself ought to suffer time-dilation. Our calculation shows that this is so for a single particle. If this is also so for a macro-object, one would have the persistence of a superposition of its spatially separated states in a frame relative to which it is moving close to the speed of light (of course, observation of its state by another macro-object at rest would rapidly collapse the statevector in that frame).

[32] John Bell, Physics 1, 195 (1964).

[33] M. Hausner and J. T. Schwartz, Lie Groups: Lie Algebras (Gordon and Breach, New York 1968), p. 57, Eq. (5). 
FIG. 1. Diagram parts: a) The vertex $2 \gamma \eta \phi$. b) The vertex $g(2 M) \phi: \psi^{2}:$ c) The "noise propagator" G attached to two $\eta$ vertices.

FIG. 2. Diagrams involved in particle production from the vacuum, for RCSL 1 . a) - c) $\phi$ particle production in order $\gamma$. d) Particle pair production in order $\gamma g^{2}$. e) $\phi$ pair production in order $\gamma g^{6}$.

FIG. 3. Diagrams responsible for collapse in (lowest) order $\gamma g^{2}$, for $\mathrm{RCSL}_{1}$.

FIG. 4. Diagrams responsible for collapse in (lowest) order $\gamma$, for $\mathrm{RCSL}_{2}$ and RCSL.

FIG. 5. Pair production diagram in order $\gamma^{2}$. 